\title{
Original paper \\ Revision of Scheumann's classification of melilitic lamprophyres and related melilitic rocks in light of new analytical data
}

\author{
Jaromír ULRYCH ${ }^{1}$, Jiří ADAMOVIČ1*, Lukáš KRMÍČEK², Lukáš ACKERMAN', Kadosa BALOGH³ \\ ${ }^{1}$ Institute of Geology, v.v.i., Academy of Sciences of the Czech Republic, Rozvojová 269, 16500 Prague 6, Czech Republic; \\ adamovic@gli.cas.cz \\ ${ }^{2}$ Brno University of Technology, Faculty of Civil Engineering, Veveři 95, 60200 Brno, Czech Republic \\ ${ }^{3}$ Institute of Nuclear Research, Hungarian Academy of Sciences, Bem tér 18/C, H-4026 Debrecen, Hungary \\ ${ }^{*}$ Corresponding author
}

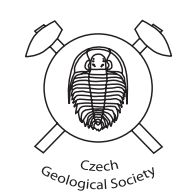

Dykes of the Late Cretaceous to Early Tertiary (79.5 \pm 3.5 to $60.7 \pm 2.4 \mathrm{Ma})$ melilitic rock series of the Osečná Complex and the Devil's Walls dyke swarm, including ultramafic lamprophyres - polzenites - of Scheumann (1913) occur dispersed in the entire Upper Ploučnice River basin in northern Bohemia.

Polzenites and associated melilitic rocks are characterized by the mineral association of olivine + melilite \pm nepheline, haüyne, monticellite, phlogopite, calcite, perovskite, spinels and apatite. New data on their mineral and chemical compositions from original Scheumann's localities (the Vesec, Modlibohov, Luhov types) argue against the abolition of the group of ultramafic lamprophyres and the terms 'polzenite' and 'alnöite' by the Le Maitre (2002) classification. Marginal facies and numerous flat apophyses of the lopolith-like body known as the Osečná Complex show an olivine micro-melilitolite composition (lamprophyric facies). The porphyritic texture, chemical composition and the presence of characteristic minerals such as monticellite and phlogopite point to their affinity with ultramafic lamprophyres - polzenites of the Vesec type. Melilite-bearing olivine nephelinites to olivine melilitites (olivine + clinopyroxene + nepheline + melilite \pm haüyne and spinels with apatite) form a swarm of subparallel dykes known as the Devil's Walls.

The Scheumann's non-melilite dyke rock "wesselite", spatially associated with polzenites and often erroneously attributed to the polzenite group, is an alkaline lamprophyre of monchiquite to camptonite composition (kaersutite + phlogopite + diopside + olivine phenocrysts in groundmass containing clinopyroxene, phlogopite, haüyne, analcime, titanian magnetite, apatite \pm glass/plagioclase). First K-Ar data show Oligocene ages (30.9 \pm 1.2 to $27.8 \pm 1.1 \mathrm{Ma}$ ) and an affinity to the common tephrite-basanite rock series.

Keywords: melilitic rocks, lamprophyres, polzenites, classification, geochemistry, Bohemian Massif

Received: 31 May 2013; accepted: 20 January 2014; handling editor: V. Rapprich

\section{Introduction}

Rare ultramafic melilite-bearing olivine nephelinite to olivine melilitite volcanic rocks and their plutonic equivalents (mostly of the melteigite-ijolite-urtite series) and associated lamprophyric alnöite represent low-volume mantle-derived melts (Ishikawa et al. 2004; Melluso et al. 2011). Rocks of melilitic volcanic series occur in intraplate rift settings (Siberia - Egorov 1970; Zhabin and Surina 1970; the Balcones Province, Texas, and Tasmania - Brey 1978; Africa - Dawson et al. 1985; Bailey et al. 2005; Keller et al. 2006; Greenland - Bernstein et al. 2000; Madagascar - Melluso et al. 2011; Euro-Mediterranean region - Lustrino and Wilson 2007; Piromallo et al. 2008 and citations therein), at continental margins (the western Canary and Madeira islands are situated on oceanic lithosphere, whereas the eastern Canary Islands on transitional, oceanic/continental lithosphere - Hoernle and Schmincke 1993) and in ocean island settings (Hawaii - Wilkinson and Stolz 1983; Maaloe et al. 1992; French Polynesia - Chauevel et al. 1992). Potassic to ultrapotassic melilitic rocks of exceptional subductionrelated orogenic settings are represented by the Roman Magmatic Province (Di Battistini et al. 2001 and citations therein) and a diatreme filling of polzenite (modlibovite) composition in California (Morgan et al. 1985).

Alnöite dykes are rare in rift-related settings, e.g., Alnö Island, Sweden (Eckermann 1961), Monteregian Hills, Canada (Hamois and Mineau 1991), MaimechaKotui Province, Siberian Platform (Egorov 1970; Zhabin and Surina 1970), Kola Peninsula (Beard et al. 1998; Rass 2008), Gardar Province, Greenland (Pearce et al. 1997), Sierra Subandinas, Argentina (Barbieri et al. 1997) but also in an island-arc setting on the Solomon Islands, Ontong Java Plateau (Ishikawa et al. 2004).

Melilitic rocks occur throughout the Central European Volcanic Province (CEVP) (Alibert et al. 1983; Keller 1984; Keller et al. 1990; Hegner et al. 1995; Wilson et al. 1995; Hegner and Vennemann 1997; Dunworth and Wilson 1998; Lustrino and Wilson 2007) including the Bohemian Massif. They are particularly common in western Bohemia and Saxony (Ulrych et al. 2000a, b; 
2010; Seifert et al. 2008; Abratis et al. 2009; Krüger et al. 2013). Besides these usual melilitic volcanic rocks with substantial clinopyroxene, Scheumann (1913, 1922) recognized clinopyroxene-free lamprophyric dyke rocks in the Ploučnice River Region (PRR) in northern Bohemia and described them as polzenites. Pivec et al. $(1986,1998)$ and Ulrych et al. $(1988,2008)$ reported the presence of the hypabyssal Osečná olivine melilitolite intrusion, also free of clinopyroxene, from the same area.

Similar Late Cretaceous flat-lying sheets of ultramafic lamprophyres are also known from other localities of the Bohemian Massif. The sheets of monchiquite-ouachitite-aillikite series penetrate the Permo-Carboniferous sediments of central Bohemia (Ulrych et al. 1993), and a phlogopite-rich olivine clinopyroxenite (autotransformed alnöite) sheet intruded the boundary between Upper Permian and Upper Cretaceous sediments near Dvůr Králové nad Labem (Ulrych et al. 1996).

The present paper summarizes information on melilitic rocks from northern Bohemia (Ulrych et al. 2008) and presents new chemical analyses and $\mathrm{K}-\mathrm{Ar}$ dating of melilitic and melilite-bearing dykes at the classical Scheumann's localities.

\section{Geological position}

Late Cretaceous to Cenozoic intraplate volcanism in Central Europe belongs to the Central European Volcanic Province (CEVP) and concentrates to the European Cenozoic Rift System (Prodehl et al. 1995). The easternmost segment of this rift system is represented by the Ohře/Eger Rift (OR) in the Bohemian Massif. Peak volcanic activity related to this structure coincided with the maximum crustal extension and graben formation in the Late Eocene to Early Miocene; however, Cenozoic volcanic products in northern Bohemian Massif are not restricted to this event. Three phases of elevated volcanic activity can be distinguished (Ulrych et al. 2011): (i) pre-rift Upper Cretaceous to Palaeocene ultramafic melilitic rock series, (ii) syn-rift Oligocene to Early Miocene tephrite/basanite-phonolite/ trachyte rock series, and (iii) sporadically occurring ultramafic rocks of the late-rift Late Miocene period.

The PRR is well known by the occurrence of Late Cretaceous to Palaeocene ultramafic intraplate volcanic rocks characterized by the presence of melilite. Scheumann (1913) defined a suite of clinopyroxene-free ultramafic lamprophyres from this region and named it after the Ploučnice River (Polzen in German) - polzenites.

The occurrences of polzenites and associated melilitic rocks in northern Bohemia concentrate along the SE marginal fault of the Ohře/Eger Rift graben. Strikes of their dykes (mostly NE-SW) are compatible with the stress field which governed the main episode of thrusting at the
NW-SE-striking Lusatian Fault (Adamovič and Coubal 1999; Ulrych et al. 2011). At this time, fractures parallel to the maximum principal stress component became dilated, permitting a rapid magma ascent through the crust.

Polzenites and associated melilitic rocks are represented by (1) dykes striking NE-SW, rarely ENE-WSW, several kilometres in length, and (2) the central intrusion of olivine melilitolite at Osečná (Pivec et al. 1986, 1998; Ulrych et al. 1988, 2008; Fig. 1). The Osečná intrusion is an asymmetrical, lopolith-shaped body, 20 to $60 \mathrm{~m}$ thick, emplaced in sediments of the Cenomanian to Turonian age (depth $0-200 \mathrm{~m}$ ) over an area of $12.5 \mathrm{~km}^{2}$ (see Fig. 2 in Ulrych et al. 2008). The dip angles of the Osečná saucer-shaped sill and flat-lying sheets are low (up to $30^{\circ}$ ) but steepen to as much as $70-80^{\circ}$ on the edges of the saucer, forming finger-like apophyses (see also similar steepening from the Whin Sill, England and Midland Valley Sill, Scotland - Francis 1982). The intrusion reaches the surface only rarely in the form of marginal facies formed by fine-grained micro-melilitolite (micro-porphyritic melilitolite).

The Osečná Complex is spatially associated with dykes of olivine nephelinite to olivine melilitite compositions, dipping steeply to the NW (Wurm 1884). These comprise (from NW to SE) the Western Devil's Wall, the Great Devil's Wall and the Lesser Devil's Wall (Fig. 1). Dykes of this composition are interspersed with a number of shorter dykes of polzenite which are of the same orientation and were obviously emplaced under the same tectonic stress field: the Kotel dyke (clinopyroxene "polzenite" alnöite) is an example. The dyke of Pelousek Hill passes the village of Modlibohov and was most probably the site of the "Modlibohov-type" polzenites of Scheumann (1913). Svárovské návrší Hill at Osečná is a segment of a cone-sheet(?) dyke dipping at moderate angles. A NWdipping micro-melilitolite dyke $700 \mathrm{~m}$ long between Vesec and Jiříčkov (Fig. 1) was mentioned by Scheumann (1913) but more attention was given to "Vesec-type" micromelilitolite dykes at Děvín and Svojkov.

Farther west, between Mimoň and Osečná, the best examples of micro-melilitolite are the Great Ralsko dyke and the morphologically prominent Děvín dyke, also dipping steeply to the NW. The NE-SW-striking alnöite dyke is located within the subsided block of the OR near Luhov (Scheumann 1913). It crosses the top of Černý vrch Hill but provides no natural outcrops. Some of the westernmost dykes, also located within the OR, include the N-dipping micro-melilitolite dyke near Svojkov known as "Bockwener Mühle" (Scheumann 1913) and the NW-dipping alnöite dyke at Brenná known as "der Damm" (Wurm 1883; Scheumann 1913).

Occurrences of olivine melilitite and polzenite dykes continue to Saxony (Zeughausgang near Hinterhermsdorf - Pfeiffer 1978 and citations therein) and to Lusatia 


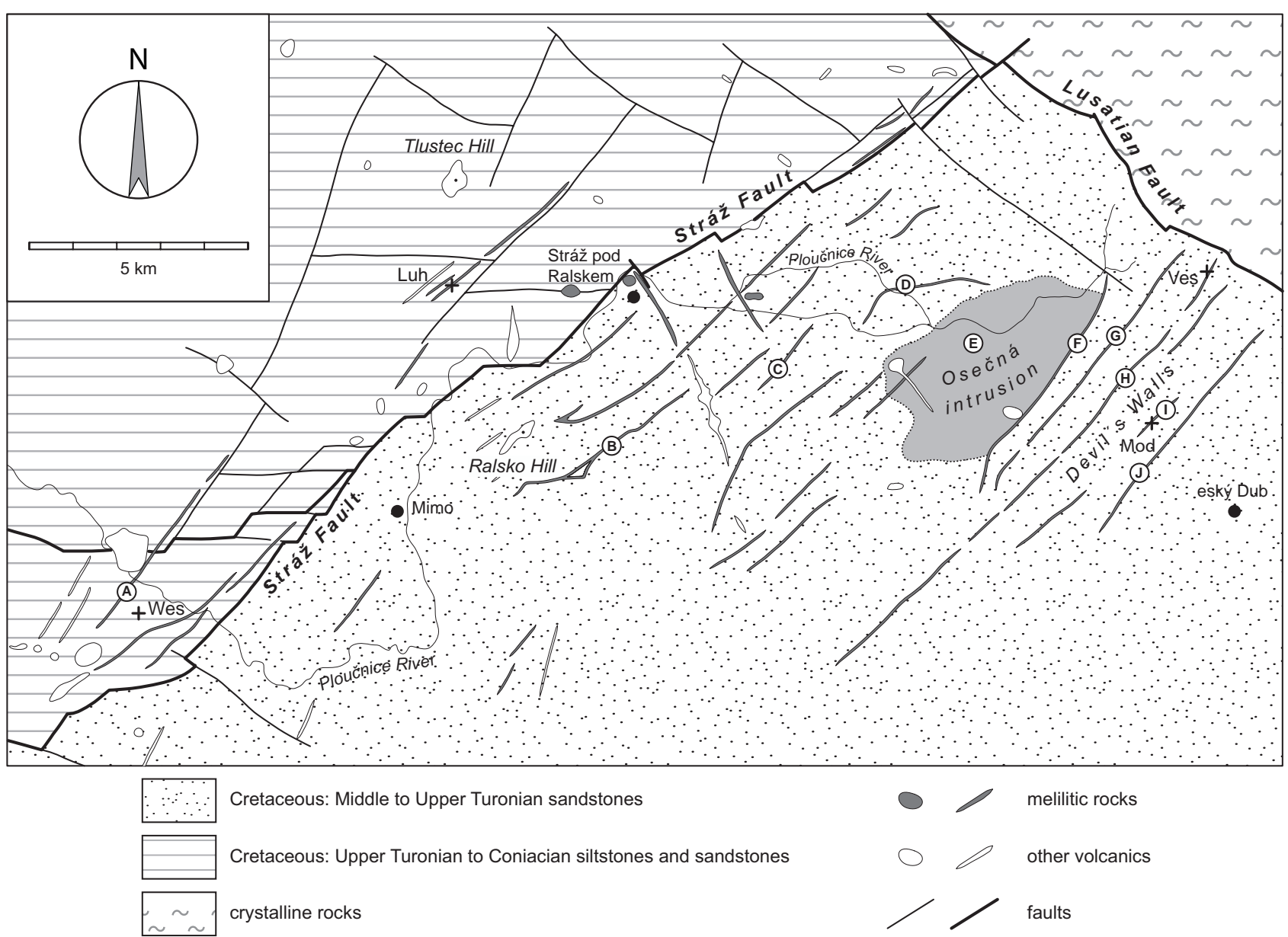

Fig. 1 A simplified geological map of northern Bohemia showing major post-Cretaceous faults and occurrences of Late Cretaceous and Cenozoic volcanic rocks in the Ploučnice River Region. Bodies of melilitic rocks: A - Brenná dyke "der Damm", B - Great Ralsko dyke, C - Děvín dyke, D - Svárovské návrší dyke, E - Osečná intrusion, F - Western Devil's Wall, G - Kotel dyke, H - Great Devil's Wall, I - Pelousek Hill dyke, J - Lesser Devil's Wall. Typical localities of melilitic rocks: Luh - luhite, Mod - modlibovite, Ves - vesecite. Wes - type locality of "wesselite" (Scheumann 1913, 1922).

(Görlitz area - Seifert et al. 2008 and citations therein). The Delitzsch Complex, occurring outside the Bohemian Massif near Leipzig, consists of Late Cretaceous ultramafic lamprophyres (alnöites and aillikites) and carbonatitic rocks (Krüger et al. 2013).

The term "wesselite" was chosen by Scheumann (1913) for rocks of the monchiquite to camptonite rock series from the "Am Knobloschen Grund" dyke at Veselí/ Wesseln SW of Mimoň. Their dykes strike mostly NNESSW in the Mimoň-Doksy area but show variable strikes further south and west. They have been reported from several places SW of studied area (Zahálka 1905; Ulrych et al. 1990, 1998; Kühn 1999).

\section{Analytical methods}

The study is based on two sets of samples. The previous dataset comes from an unpublished report for the
Uranium Industry Company and included major-element analyses (Ulrych et al. 1990). Trace-element concentrations of this sample set were acquired within the present study. The newly analysed set of samples comes from Scheumann's type localities and other outcrops in the area.

New bulk analyses followed the methods described in detail by Johnson and Maxwell (1981) and Potts (1995) and included total digestion of the sample using HF$\mathrm{HClO}_{4}$ attack and/or alkaline sintering (both in Pt dishes and/or crucibles) and subsequent determination of majorelement oxides by volumetric analyses $\left(\mathrm{Al}_{2} \mathrm{O}_{3}, \mathrm{Fe}_{2} \mathrm{O}_{3}\right.$, $\mathrm{FeO}, \mathrm{MgO}, \mathrm{CaO}$ ), spectrophotometric and/or inductively coupled plasma spectrometry (ICP-OES) analyses $\left(\mathrm{P}_{2} \mathrm{O}_{5}\right.$ and $\left.\mathrm{TiO}_{2}\right)$, and gravimetric analyses $\left(\mathrm{SiO}_{2}, \mathrm{H}_{2} \mathrm{O}\right)$. The $\mathrm{Na}_{2} \mathrm{O}, \mathrm{K}_{2} \mathrm{O}$ and $\mathrm{MnO}$ contents were determined using flame atomic absorption spectrometry (FAAS) and/or ICP-OES. The accuracy was monitored analysing the BCR-2 reference material BCR-2 (USGS) and yielded 
an average error $(1 \sigma)$ less than $\pm 5 \%$ for all determined elements.

Bulk analyses of the previous set of samples were performed at the Faculty of Science, Charles University, Prague, using the methods described in detail above.

Trace-element concentrations in the new set of samples were determined using quadrupole-based ICP-MS X Series 2 (Faculty of Science, Charles University, Prague). Those in the previous set were determined on sector-field ICP-MS Thermo Element 2 at Institute of Geology, Academy of Sciences of the Czech Republic. The analytical protocol for both sets followed the methods of Strnad et al. (2005). In brief, 0.1-0.2 $\mathrm{g}$ of sample powder were decomposed in a mixture of $\mathrm{HF}-\mathrm{HClO}_{4}$ acids in teflon beakers and/or Pt crucibles. The remaining residue was thereafter dissolved using $2 \% \mathrm{HNO}_{3}$ and transferred to $100 \mathrm{ml}$ volumetric flask. This solution was finally diluted for ICP-MS measurements. Instrumental calibration was performed using an aqueous multi-element calibration solutions and ${ }^{115} \mathrm{In}$ as an internal standard for the correction of instrumental drift. The precision of the analyses was always better than $\pm 10 \%(2 \sigma)$ for X Series 2 ICP-MS and $\pm 5 \%$ for Element 2 ICP-MS. The accuracy of $\pm 5 \%$ $(2 \sigma)$ was obtained comparing the long-term reproducibility of the USGS reference material BCR-2 against the recommended values (Jochum and Nohl 2008).

The $\mathrm{K}-\mathrm{Ar}$ age determinations were carried out according to the procedure described in Balogh (1985). Data were calibrated by interlaboratory standards LP-6, GL-O, HD-B1 and Asia 1/65, as well as atmospheric Ar (Odin et al. 1982).

$\mathrm{The} \mathrm{Sr}-\mathrm{Nd}$ isotope compositions were determined in the isotope laboratory at Universität München (Germany) according to the procedures outlined by Hegner et al. (1995). The ${ }^{143} \mathrm{Nd} /{ }^{144} \mathrm{Nd}$ ratios were determined with a Finnigan MAT 261 using a dynamic triple mass method and monitoring ${ }^{147} \mathrm{Sm}$; Sm isotopes were determined in a static data collection mode. The ${ }^{143} \mathrm{Nd} /{ }^{144} \mathrm{Nd}$ ratios were normalized to ${ }^{146} \mathrm{Nd} /{ }^{144} \mathrm{Nd}=0.7219$ and ${ }^{147} \mathrm{Sm} /{ }^{152} \mathrm{Sm}=$ 0.56081 . The ${ }^{143} \mathrm{Nd} /{ }^{144} \mathrm{Nd}$ ratio of the in-house Ames $\mathrm{Nd}$ standard solution was $0.512142 \pm 12(\mathrm{n}=35)$, corresponding to 0.511854 in the La Jolla Nd reference material. The $\varepsilon \mathrm{Nd}(\mathrm{t})$ values were calculated with the parameters of Jacobsen and Wasserburg (1980). Present-day ratios for the chondrite uniform reservoir (CHUR) were: ${ }^{147} \mathrm{Sm} /{ }^{144} \mathrm{Nd}=$
$0.1967,{ }^{143} \mathrm{Nd} /{ }^{144} \mathrm{Nd}=0.512638($ Jacobsen and Wasserburg $1980 ;{ }^{143} \mathrm{Nd} /{ }^{144} \mathrm{Nd}$ re-normalized to $\left.{ }^{146} \mathrm{Nd} /{ }^{144} \mathrm{Nd}=0.7219\right)$. The ${ }^{87} \mathrm{Sr} /{ }^{86} \mathrm{Sr}$ ratios were determined by a dynamic double mass method, monitoring ${ }^{85} \mathrm{Rb}$, and normalized to ${ }^{86} \mathrm{Sr} /{ }^{88} \mathrm{Sr}$ $=0.1194$. The NIST 987 reference material yielded ${ }^{87} \mathrm{Sr} /{ }^{86} \mathrm{Sr}=0.710230 \pm 11(\mathrm{n}=22)$.

\section{Polzenite classification and petrographic characteristics}

Scheumann (1913) recognized two types of clinopyroxene-free lamprophyres - polzenites - in the PRR: Vesec type (type locality of Vesec) and Modlibohov type (type locality of Modlibohov/Modlibov - Pelousek Hill). The original analyses of the Vesec type, however, came from Malý Bor-Svojkov/Klein Haida-Schwojka and Děvín/ Děvin Hill near Hamr (Děvin type). Scheumann (1922) additionally distinguished the third type of "polzenite" Luhov type (type locality of Luhov/Luh near Stráž pod Ralskem) which, however, corresponds to alnöite.

Of the above mentioned local names, only the general term polzenite was used in some classifications of lamprophyres (Streckeisen 1979; Le Maitre 1989; Rock 1991). Nonetheless, its understanding is often hindered by insufficient comprehension of the original German text (Scheumann 1913, 1922). In the recent classifications of Woolley et al. (1996) and Le Maitre (2002), terms like polzenite, alnöite and even the group of ultramafic lamprophyres are abolished, and melilitic lamprophyres are classified among common melilitic rocks (see the criticism by Tappe et al. 2005). Based on Tappe et al. (2005), we present a proposal of classification of ultramafic lamprophyres, including polzenites from the original localities (Tab. 1).

\subsection{Ultramafic lamprophyres and associated melilitic and melilite-bearing volcanic rocks}

\subsubsection{Ultramafic lamprophyres}

The original Scheumann's definition of melilitic lamprophyre - polzenite (Scheumann 1913) emphasized its dyke form, porphyritic to doleritic texture, and in

Tab. 1 Proposed classification of ultramafic lamprophyres based on their modal compositions

\begin{tabular}{|c|c|c|c|c|c|c|c|c|c|}
\hline \multirow[b]{2}{*}{ Rock } & \multicolumn{5}{|c|}{ Ground mas s } & \multicolumn{4}{|c|}{ Phenocrysts } \\
\hline & Melilite & Nepheline & $\begin{array}{l}\text { Sodalite } \\
\text { group }\end{array}$ & $\begin{array}{l}\text { Alkali } \\
\text { feldspar }\end{array}$ & Carbonate & Clinopyroxene & Phlogopite & Olivine & Amphibole \\
\hline Polzenite & $\mathrm{MN}$ & $\mathrm{M}$ & $\mathrm{m}$ & - & $\mathrm{m}$ & - & $\mathrm{M} /$ also in $\operatorname{grdm}$ & $\mathrm{M}$ & - \\
\hline Alnöite & $\mathrm{MN}$ & $\mathrm{m}$ & & & $\mathrm{m}$ & $\mathrm{M} /$ also in grdm $\mathrm{N}$ & $\mathrm{M} /$ also in $\operatorname{grdm} \mathrm{N}$ & M & - \\
\hline Aillikite & - & - & - & - & $\mathrm{MN}$ & M & $\mathrm{M} /$ also in grdm & M & $\mathrm{M} /$ also in grdm \\
\hline Damtjernite & - & $\mathrm{mN}^{*}$ & - & $\mathrm{mN}^{*}$ & $\mathrm{~m}$ & M/also in grdm & $\mathrm{M} /$ also in grdm & - & - \\
\hline
\end{tabular}

$\mathrm{M}$ - major constituent, $\mathrm{m}$ - minor constituent, $\mathrm{N}$ - necessary, presence of only one "asterisk" phase, - absent, grdm - groundmass 
particular, the absence of clinopyroxene and feldspar, but the presence of haüyne and phlogopite (for modal composition of polzenites see tab. 1 in Pivec et al. 1998). Polzenite is thus a counterpart of the clinopyroxenebearing melilitic, foid-free lamprophyric dyke rock - alnöite, defined by Rosenbusch (1887). The basic mineral association of polzenite is: olivine + melilite + nepheline + phlogopite + spinel + calcite \pm monticellite, haüyne (clinopyroxene), perovskite and apatite. Scheumann (1922) described the polzenite series formed by olivinerich polzenite of the Vesec type, transitional member of the Modlibohov type, to olivine-free bergalite from Kaiserstuhl (Söllner 1913). A characteristic macroscopic feature of polzenites is the warty surface (Fig. 2a).

The Vesec type (vesecite) of polzenite was defined by Scheumann (1913) as a rock rich in olivine with monticellite rims and unevenly distributed phlogopite (Fig. 2b). The micro-porphyritic texture is characteristic; olivine and phlogopite micro-phenocrysts $(0.1-0.8 \mathrm{~mm})$ are set in a fine-grained groundmass with trachytic texture.

The Modlibohov type (modlibovite) of Scheumann (1913) represents an olivine- and phlogopite-rich rock with zoned foids (haüyne core and sodalite rim: Ulrych et al. 1991). The texture is micro-porphyritic to doleritic with olivine and phlogopite $(0.1-0.8 \mathrm{~mm})$ microphenocrysts set in trachytic and poikilitic groundmass (Fig. 2c). Rare irregular clusters of clinopyroxene grains were detected in some modlibovite samples.

The Luhov type (luhite) is compositionally and texturally similar to modlibovite (Scheumann 1922) containing also minerals of the sodalite group (Ulrych et al. 1991). However, it has variable amounts of clinopyroxene micro-phenocrysts (up to 20 vol. $\% ; 0.2-1 \mathrm{~mm}$ ) forming irregular rims around olivine in association with carbonate (Fig. 2d). Scheumann (1922) classified it to haüyne-melilite damtjernite. The Luhov type thus belongs to alnöite and not to polzenite (Johannsen 1949; Le Maitre 1989).

Ultramafic melilitic lamprophyres of the PRR are accompanied by other melilitic magmatic rocks forming (i) a hypabyssal olivine melilitolite sheet beneath Osečná and (ii) a swarm of melilite-bearing olivine nephelinite to olivine melilitite dykes (the Devil's Walls swarm).

\subsubsection{Olivine melilitolite and olivine micro- -melilitolite of the Osečná Complex}

The lopolith-like sheet of the Osečná Complex is not strictly homogeneous. It is a hypabyssal equivalent of near-surface dykes. The central part is composed of olivine melilitolite: olivine + melilite + spinels \pm nepheline, phlogopite, monticellite, perovskite and carbonate. The olivine melilitolite is a medium-grained porphyritic rock of lamprophyric character. Phenocrysts are formed by olivine, melilite and phlogopite $(1-5 \mathrm{~mm})$ set in groundmass with ophitic to poikiloophitic texture (see photo 2 in Ulrych et al. 1988).

The intrusion contains also rare dykes and pods of coarse-grained rocks such as melilitolite pegmatoids, ijolites, and glimmerites. They gradually pass into the parental olivine melilitolite (Ulrych et al. 2008).

The outermost parts of the melilitolite intrusion formed by its chilled margins and numerous apophyses are composed of olivine micro-melilitolite with microphenocrysts of olivine set in groundmass with trachytoid texture. Phenocrysts are formed by olivine with monticellite rims and phlogopite. The groundmass consists of melilite and nepheline \pm sodalite-haüyne \pm carbonate; spinels, perovskite and apatite are common accessory minerals. The mineral association and textural characteristics of olivine micro-melilitolite are very similar to those of dykes of ultramafic lamprophyres - polzenites - of the Vesec type.

\subsubsection{Melilite-bearing olivine nephelinite (melanephelinite) to olivine melilitite of the Devil's Walls}

The Devil's Walls dykes are composed of rocks of microporphyritic melilite-bearing olivine nephelinite to rare olivine melilitite series. The microphenocrysts (0.1-1 mm) are composed of olivine and clinopyroxene, while the fine-grained holocrystalline groundmass (see photo 10 in Ulrych et al. 1998) consists of nepheline, melilite, olivine, clinopyroxene, phlogopite, spinels, apatite and rare perovskite.

\subsection{Alkaline lamprophyres}

Scheumann (1913) described the melilite-free lamprophyre as biotite-haüyne basalt of the monchiquite and bekinkinite type, i.e., a melanocratic variety of theralite of Rosenbusch (1907), from "Am Knobloschen Grund" dyke at Veselí/Wesseln. The herein verified mineral association of this alkaline lamprophyre of coarse-porphyritic texture and holocrystalline to hemicrystalline groundmass from the original locality of Veselí ("wesselite") is kaersutite (Fig. 2f) + titanian phlogopite + diopside + (serpentinized) olivine phenocrysts $(1-10 \mathrm{~mm})$ in groundmass containing clinopyroxene, phlogopite, haüyne, analcime, titanian magnetite and apatite cemented by weakly anisotropic matter $\left(2.4\right.$ wt. $\% \mathrm{Na}_{2} \mathrm{O}, 3.5$ wt. \% $\mathrm{K}_{2} \mathrm{O}, 0.5$ wt. $\% \mathrm{SO}_{3}, 12.5$ wt. $\% \mathrm{CO}_{2}$ ). The origin of the latter by decomposition of foids and/or glass is most probable. No melilite or its alteration products were identified. By modal composition, this rock corresponds to monchiquite (Le Maitre 2002). The modal composition of the rock is rather variable probably due to the zoning 

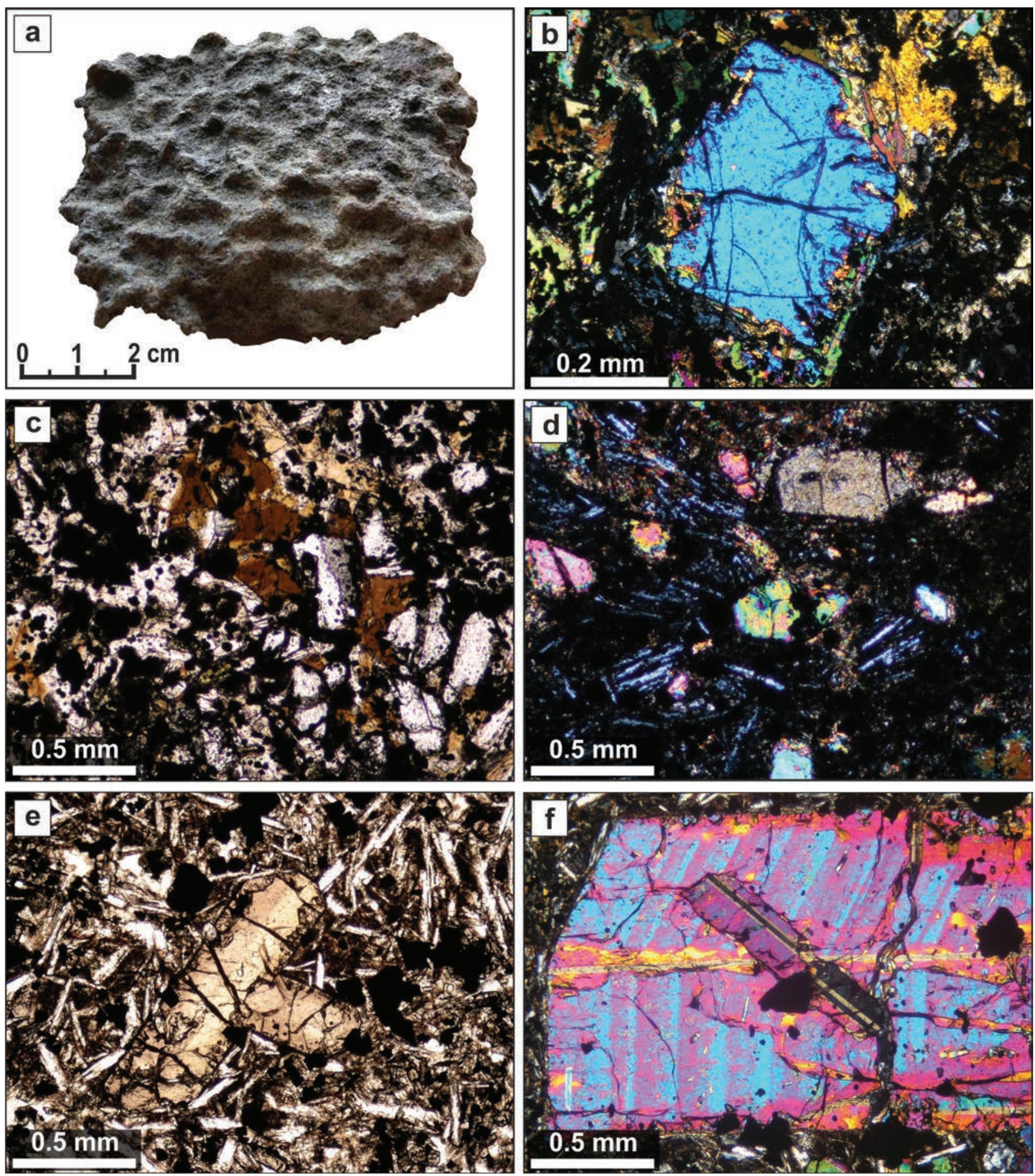

Fig. 2 Petrographic features of the studied lamprophyric rocks (photomicrographs: PPL = plane-polarized light; XPL = cross-polarized light). $\mathbf{a}$ - Characteristic warty surface of polzenites (locality Vesec); b - olivine with monticellite rim in polzenite of the Vesec type (locality Vesec; XPL); $\mathbf{c}$ - micro-porphyritic texture of the Modlibohov type polzenite characterized by olivine phenocrysts in a groundmass containing poikilitic phlogopite (locality Modlibohov; PPL); d - clinopyroxene-rich "polzenite" - alnöite of the Luhov type with laths of melilite-group minerals in groundmass (locality Luhov; XPL); $\mathbf{e}$ - texture of "wesselite" (camptonite) characterized by phenocrysts of kaersutite surrounded by pilotaxitic feldspar laths of groundmass (locality Veselí; PPL); $\mathbf{f}$ - twinned kaersutite crystal enclosed in a larger, strongly zoned grain of the same mineral ("wesselite", locality Veselí; XPL). 
of the dyke or a presence of several subparallel dykes. It manifests in particular in the presence of plagioclase or glass/analcime. The monchiquite from Veselí (samples 49A and 1/13) and camptonites with a low proportion of ternary labradorite to andesine (Ulrych et al. 1998) from the Střelnice dyke at Doksy (sample X-1) and Liščí vrch Hill near Doksy (sample 42) are very similar in composition. Nevertheless, those from Veselí P-1* (Fig. 2e) and Svojkov (sample 35) represent the leuco-camptonite variety, and sample 49 is a mela-camptonite.

Scheumann (1922) assigned "wesselite" to monchiquite but associated it within the same rock series as polzenites. However, Tröger (1939), Wimmenauer (1973) and Rock (1991) classified "wesselite" directly to polzenites in spite of the absence of melilite.

\section{Geochemical characteristics}

\subsection{Ultramafic lamprophyres, olivine melilitolite, and associated melilitic and melilite-bearing volcanic rocks}

Concise geochemical characteristics of melilitic and melilite-bearing rocks of the PRR were presented by Ulrych et al. (1988, 2008) and Pivec at al. (1998). However, new geochemical and $\mathrm{K}-\mathrm{Ar}$ geochronological data on polzenites from Scheumann's original localities, and the associated specific alkaline lamprophyre "wesselite" (Ulrych et al. 1990) from this region have not been published yet (Tab. 2). A comparison of geological and, in particular, mineralogical, petrographic and geochemical characteristics of the individual melilitic and melilite-bearing rocks is presented in Tab. 3.

Lamprophyric dyke rocks, including hypabyssal olivine melilitolite, are characterized by very low $\mathrm{SiO}_{2}$ and alkalis, while $\mathrm{CaO}$ and $\mathrm{MgO}$ contents are very high (Tab. 2). Melilite-bearing olivine nephelinite to olivine

Fig. 3 Total alkali-silica (TAS) diagram (Le Maitre 2002) for the Late Cretaceous and Cenozoic volcanic rocks of the Ploučnice River Region. Data from Ulrych et al. (2008) and this work. Melilitic and melilite-bearing rock group include olivine melilitolite, olivine melilitolite pegmatoid, ijolite, olivine micro-melilitolite, melilite-bearing olivine nephelinite and olivine melilitite. melilitite is partly richer in $\mathrm{SiO}_{2}, \mathrm{Al}_{2} \mathrm{O}_{3}$ and alkalis but poorer in $\mathrm{CaO}$, and $\mathrm{MgO}$ (see Tab. 2). Individual types of the melilitic and melilite-bearing rocks plot in the foidite field of the TAS diagram of Le Maitre (2002) (Fig. 3). All samples of these rocks, with the exception of the melilitebearing olivine nephelinite, are larnite-normative (CIPW norm) and thus can be classified as olivine melilitite sensu Brey (1978). In the $\mathrm{CaO}+\mathrm{Na}_{2} \mathrm{O}+\mathrm{K}_{2} \mathrm{O}$ wt. \% vs. $\mathrm{SiO}_{2}+\mathrm{Al}_{2} \mathrm{O}_{3}$ wt. \% diagram of Le Bas (1989), melilitebearing rocks mostly fall into the melilitite field (Fig. 4).

According to the criteria of Frey et al. (1978), primary melts of mantle origin should have: (i) $\mathrm{Mg} \#[\mathrm{Mg} \#=100$ $\mathrm{Mg} /\left(\mathrm{Mg}+\mathrm{Fe}^{2+}\right)$ where $\mathrm{Fe}^{2+}$ is calculated on the basis of $\mathrm{Fe}^{3+} \mathrm{Fe}=0.15$ ] between 68 and 75, (ii) high contents of compatible elements such as $\mathrm{Ni}(>300 \mathrm{ppm})$, Co $(>50$ $\mathrm{ppm}), \mathrm{Sc}(>30 \mathrm{ppm})$, and $\mathrm{Cr}(>600 \mathrm{ppm})$ and (iii) upper mantle xenoliths. Based on these criteria, the melilitic and melilite-bearing volcanic rocks of the PRR represent near-primary melts $(\mathrm{Mg} \#=73-81, \mathrm{Ni}=\sim 130-670$ $\mathrm{ppm}, \mathrm{Co}=\sim 50-70 \mathrm{ppm}, \mathrm{Sc}=\sim 20-40 \mathrm{ppm}$ and $\mathrm{Cr}=$ $\sim 140-1300 \mathrm{ppm}$ ) in equilibrium with the primitive mantle peridotite which underwent only limited low-pressure fractional crystallization (Brey 1978; Alibert et al. 1983). Melilitic rocks have also high $\mathrm{CaO} / \mathrm{Al}_{2} \mathrm{O}_{3}$ ratios (1.3-2.4), thus exceeding those of typical ocean island and midocean ridge basalts (OIB and MORB) which are both less than unity (Sun and McDonough 1989).

The strongest alteration effects are seen within the Osečná olivine melilitolite intrusion. The following types

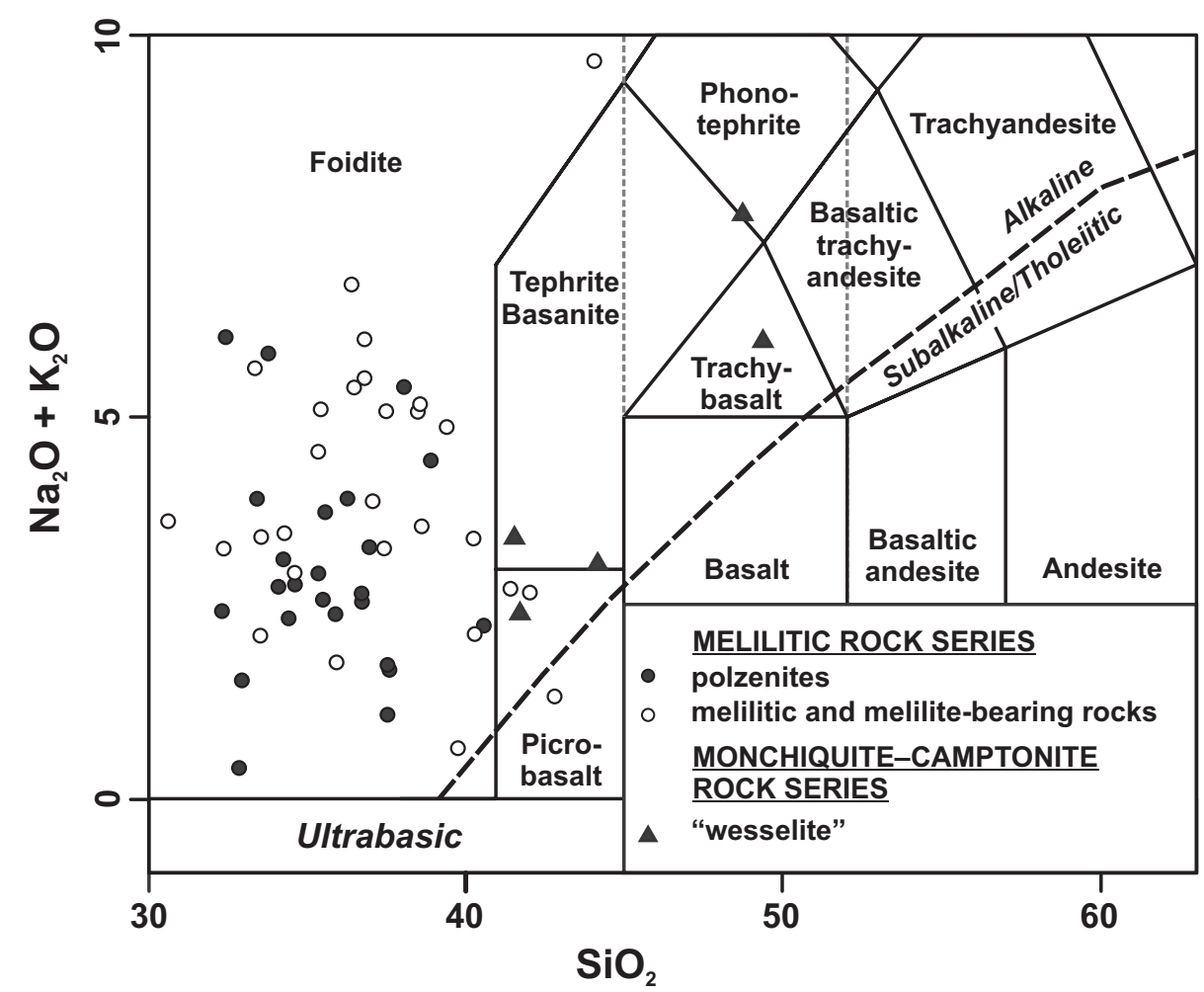


Tab. 2 Representative whole-rock major- and trace-element analyses of the melilitic and monchiquite-camptonite rock series of the Ploučnice River Region

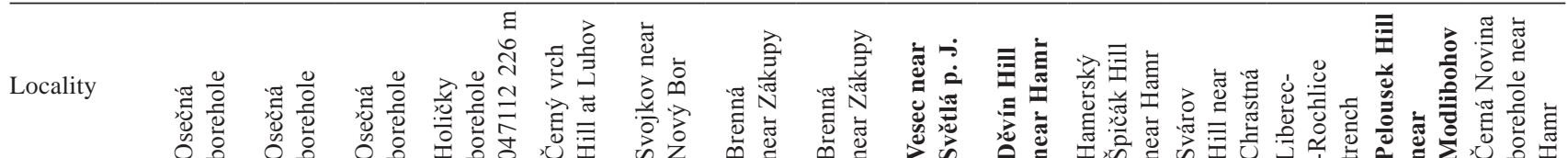

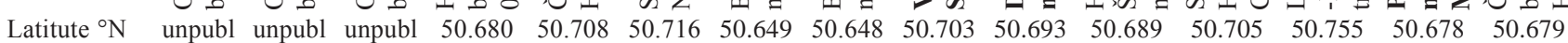

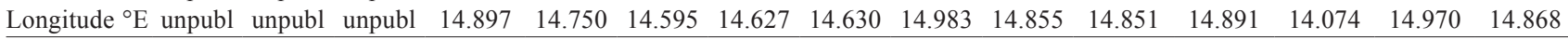
\begin{tabular}{llllllllllllllll}
\hline Sample & $87 \mathrm{~B}$ & 88 & 8 & $\mathrm{P}-12 *$ & $25 \mathrm{~A}$ & 33 & 55 & 56 & $\mathrm{P}-9 *$ & 57 & 58 & $22 \mathrm{~B}$ & 36 & $\mathrm{P}-2 *$ & 65
\end{tabular}

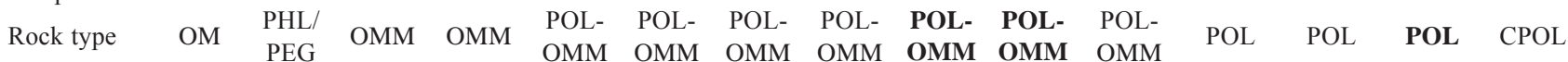

\begin{tabular}{|c|c|c|c|c|c|c|c|c|c|c|c|c|c|c|c|}
\hline$\overline{\mathrm{SiO}_{2} \text { (wt. \%) }}$ & 32.09 & 34.36 & 31.15 & 31.50 & 31.03 & 31.75 & 34.02 & 34.32 & 30.32 & 32.84 & 32.33 & 33.07 & 38.17 & 35.02 & 32.60 \\
\hline $\mathrm{TiO}_{2}$ & 2.84 & 1.74 & 2.28 & 2.57 & 2.13 & 2.20 & 2.08 & 2.17 & 1.97 & 2.23 & 2.23 & 2.36 & 2.36 & 2.10 & 2.75 \\
\hline $\mathrm{Al}_{2} \mathrm{O}_{3}^{2}$ & 8.24 & 6.84 & 8.13 & 8.65 & 8.15 & 8.42 & 7.57 & 8.92 & 7.61 & 8.71 & 8.25 & 8.21 & 10.02 & 8.68 & 6.62 \\
\hline $\mathrm{Fe}_{2}^{2} \mathrm{O}_{3}^{3}$ & 5.61 & 3.83 & 6.21 & 7.57 & 6.69 & 6.44 & 4.16 & 4.05 & 7.15 & 4.85 & 4.31 & 7.46 & 4.43 & 6.03 & 3.52 \\
\hline $\mathrm{FeO}^{3}$ & 5.63 & 5.74 & 6.49 & 2.81 & 4.99 & 4.06 & 5.99 & 6.00 & 4.29 & 5.68 & 6.07 & 3.86 & 6.11 & 5.41 & 6.00 \\
\hline $\mathrm{MnO}$ & 0.23 & 0.22 & 0.17 & 0.17 & 0.19 & 0.18 & 0.16 & 0.16 & 0.20 & 0.18 & 0.18 & 0.20 & 0.18 & 0.22 & 0.17 \\
\hline $\mathrm{MgO}$ & 14.54 & 7.92 & 15.87 & 13.38 & 16.60 & 16.04 & 18.35 & 15.79 & 17.99 & 17.11 & 17.24 & 15.81 & 13.49 & 15.27 & 19.12 \\
\hline $\mathrm{CaO}$ & 19.47 & 22.01 & 17.65 & 17.89 & 18.05 & 17.90 & 15.25 & 16.74 & 20.53 & 19.26 & 18.82 & 16.53 & 15.48 & 18.38 & 15.78 \\
\hline $\mathrm{Na}_{2} \mathrm{O}$ & 3.01 & 3.09 & 2.13 & 0.22 & 4.41 & 0.96 & 0.92 & 0.81 & 0.47 & 2.17 & 1.47 & 2.30 & 1.91 & 1.76 & 1.65 \\
\hline $\mathrm{K}_{2} \mathrm{O}$ & 2.78 & 3.51 & 1.59 & 1.98 & 1.70 & 2.20 & 1.31 & 1.40 & 0.70 & 1.41 & 1.39 & 1.71 & 0.85 & 1.39 & 1.17 \\
\hline $\mathrm{P}_{2}^{2} \mathrm{O}_{5}$ & 1.67 & 5.11 & 1.01 & 0.95 & 1.73 & 1.52 & 0.87 & 0.95 & 1.02 & 1.46 & 1.60 & 1.52 & 1.07 & 1.10 & 1.32 \\
\hline $\mathrm{H}_{2}^{2} \mathrm{O}^{+}$ & 2.66 & 4.17 & 2.14 & 4.66 & 3.37 & 5.95 & 6.33 & 6.16 & 5.78 & 2.69 & 3.50 & 6.02 & 3.56 & 3.34 & 2.60 \\
\hline $\mathrm{H}_{2}^{2} \mathrm{O}^{-}$ & 0.04 & 0.10 & 0.66 & 1.92 & 0.26 & 0.54 & 0.98 & 0.85 & 0.32 & 0.33 & 0.20 & 0.26 & 0.84 & 0.20 & 1.50 \\
\hline $\mathrm{CO}_{2}$ & 0.61 & 0.87 & 4.09 & 5.39 & 0.81 & 1.17 & 1.60 & 0.93 & 1.02 & 0.50 & 1.23 & 0.91 & 1.42 & 0.44 & 4.52 \\
\hline $\mathrm{F}_{2}$ & 0.23 & 0.44 & 0.12 & n.d. & 0.13 & 0.21 & 0.16 & 0.26 & n.d. & 0.20 & 0.20 & 0.15 & 0.16 & n.d. & 0.37 \\
\hline $\mathrm{S}^{2}$ & n.d & n.d & 0.16 & n.d. & 0.26 & 0.08 & 0.11 & 0.17 & n.d. & 0.10 & 0.13 & 0.09 & 0.10 & n.d. & n.d \\
\hline Total & 99.65 & 99.95 & 99.85 & 99.74 & 100.50 & 99.62 & 99.86 & 99.68 & 99.62 & 99.72 & 99.15 & 100.46 & 100.15 & 99.64 & 99.69 \\
\hline $\mathrm{Ni}(\mathrm{ppm})$ & 280 & 74 & 277 & 216 & 367 & 345 & 470 & 336 & 276 & 460 & 302 & 355 & 265 & 321 & 332 \\
\hline $\mathrm{Cr}$ & 512 & 25 & 785 & 700 & 596 & 463 & 788 & 625 & 800 & 885 & 430 & 645 & 556 & 559 & 497 \\
\hline Co & 48.7 & 31.2 & 58.4 & 47.0 & 62.3 & 66.3 & 61.9 & 52.0 & 61.0 & 62.0 & 55.6 & 61.3 & 60.9 & 56.0 & 59.6 \\
\hline $\mathrm{Sc}$ & 32.9 & 56.1 & 34.0 & 21.8 & 32.3 & 44.5 & 26.2 & 29.6 & 25.5 & 38.5 & 32.0 & 31.2 & 33.0 & 29.9 & 26.0 \\
\hline $\mathrm{Rb}$ & 56.8 & 121 & 53.7 & 65.0 & 45.8 & 55.1 & 44.4 & 50.5 & 25.0 & 46.3 & 46.4 & 57.8 & 51.6 & 45.0 & 36.3 \\
\hline $\mathrm{Sr}$ & 2012 & 2513 & 2046 & 1277 & 1764 & 1225 & 1741 & 2246 & 1897 & 1522 & 1226 & 1269 & 1770 & 2225 & 1110 \\
\hline $\mathrm{Y}$ & 40 & 71 & 29 & 29 & 31 & 35 & 24 & 28 & 27 & 26 & 28 & 33 & 37 & 41 & 20 \\
\hline $\mathrm{Zr}$ & 758 & 855 & 307 & 345 & 263 & 373 & 260 & 325 & 271 & 242 & 233 & 314 & 308 & 379 & 155 \\
\hline $\mathrm{Nb}$ & 187 & 79 & 184 & 125 & 154 & 164 & 124 & 144 & 149 & 189 & 157 & 140 & 137 & 179 & 121 \\
\hline Cs & 1.03 & 2.34 & 5.46 & 3.03 & 0.79 & 0.60 & 0.86 & 1.04 & 0.46 & 0.89 & 0.97 & 1.00 & 1.19 & 0.74 & 1.89 \\
\hline $\mathrm{Ba}$ & 959 & 1391 & 1138 & 754 & 634 & 917 & 680 & 922 & 541 & 1459 & 1017 & 731 & 827 & 2115 & 698 \\
\hline $\mathrm{La}$ & 119 & 26.4 & 127 & 83.6 & 127 & 141 & 94.9 & 98.1 & 150 & 99.9 & 109 & 115 & 107 & 158 & 85.6 \\
\hline $\mathrm{Ce}$ & 220 & 31.0 & 246 & 177 & 235 & 252 & 178 & 179 & 300 & 182 & 201 & 219 & 200 & 280 & 164 \\
\hline $\mathrm{Pr}$ & 24.8 & 3.1 & 27.8 & 19.9 & 26.0 & 27.4 & 19.3 & 19.4 & 31.0 & 20.8 & 22.3 & 24.7 & 22.2 & 27.5 & 18.4 \\
\hline $\mathrm{Nd}$ & 92.6 & 11.8 & 10.3 & 77.2 & 95.2 & 98.8 & 73.6 & 73.5 & 110 & 80.1 & 84.5 & 91.0 & 81.5 & 98.3 & 71.5 \\
\hline $\mathrm{Sm}$ & 15.7 & 3.0 & 16.7 & 13.4 & 15.4 & 15.5 & 12.0 & 12.3 & 17.2 & 12.9 & 14.1 & 15.0 & 13.1 & 16.5 & 11.8 \\
\hline $\mathrm{Eu}$ & 4.80 & 1.66 & 5.10 & 3.85 & 4.59 & 4.76 & 3.62 & 3.85 & 4.87 & 3.74 & 4.27 & 4.45 & 3.73 & 4.94 & 3.51 \\
\hline $\mathrm{Gd}$ & 14.3 & 5.2 & 14.8 & 13.9 & 13.8 & 14.0 & 10.7 & 11.2 & 18.0 & 10.3 & 12.5 & 13.6 & 11.6 & 18.3 & 10.2 \\
\hline $\mathrm{Tb}$ & 1.88 & 0.90 & 1.81 & 1.62 & 1.73 & 1.75 & 1.34 & 1.44 & 1.82 & 1.28 & 1.58 & 1.67 & 1.48 & 2.10 & 1.25 \\
\hline Dy & 9.33 & 7.15 & 8.56 & 6.28 & 8.12 & 8.36 & 6.39 & 7.13 & 6.35 & 6.44 & 7.55 & 8.25 & 7.25 & 8.38 & 5.81 \\
\hline Ho & 1.61 & 1.77 & 1.27 & 0.97 & 1.24 & 1.33 & 1.00 & 1.16 & 0.97 & 1.07 & 1.19 & 1.28 & 1.19 & 1.41 & 0.86 \\
\hline $\mathrm{Er}$ & 4.04 & 6.36 & 3.10 & 2.87 & 3.02 & 3.29 & 2.45 & 2.82 & 2.83 & 2.58 & 2.84 & 3.15 & 3.02 & 3.93 & 2.07 \\
\hline $\mathrm{Tm}$ & 0.56 & 0.99 & 0.31 & 0.29 & 0.31 & 0.37 & 0.26 & 0.32 & 0.25 & 0.31 & 0.30 & 0.35 & 0.36 & 0.42 & 0.19 \\
\hline $\mathrm{Yb}$ & 3.11 & 7.15 & 1.69 & 1.82 & 1.72 & 2.03 & 1.48 & 1.77 & 1.57 & 1.80 & 1.67 & 1.88 & 1.99 & 2.54 & 1.15 \\
\hline $\mathrm{Lu}$ & 0.53 & 1.06 & 0.22 & 0.25 & 0.22 & 0.29 & 0.19 & 0.19 & 0.18 & 0.25 & 0.21 & 0.25 & 0.28 & 0.33 & 0.13 \\
\hline Hf & 12.70 & 13.30 & 5.55 & 7.77 & 4.61 & 6.15 & 4.61 & 5.94 & 5.75 & 5.56 & 4.32 & 5.20 & 5.19 & 8.67 & 2.93 \\
\hline $\mathrm{Ta}$ & 7.33 & 0.26 & 8.96 & 7.73 & 7.06 & 6.15 & 5.90 & 6.35 & 8.91 & 3.65 & 6.34 & 6.47 & 5.85 & 8.11 & 6.51 \\
\hline Th & 11.8 & 0.33 & 14.5 & 10.2 & 12.7 & 14.7 & 10.7 & 10.6 & 19.8 & 12.1 & 11.9 & 10.6 & 15.1 & 19.5 & 9.41 \\
\hline $\mathrm{U}$ & 4.77 & 3.21 & 3.25 & 2.49 & 3.16 & 4.11 & 2.24 & 2.83 & 3.76 & 3.87 & 3.06 & 3.03 & 3.37 & 5.63 & 2.34 \\
\hline$\overline{\mathrm{Mg} \#}$ & 74.1 & 64.4 & 73.4 & 74.5 & 76.0 & 77.4 & 79.8 & 77.5 & 77.9 & 78.1 & 78.4 & 75.8 & 73.7 & 74.7 & 81.4 \\
\hline $\mathrm{K} / \mathrm{Rb}$ & 406 & 241 & 246 & 253 & 308 & 331 & 245 & 230 & 232 & 253 & 249 & 246 & 137 & 256 & 268 \\
\hline $\mathrm{Rb} / \mathrm{Sr}$ & 0.028 & 0.048 & 0.026 & 0.051 & 0.026 & 0.045 & 0.026 & 0.022 & 0.013 & 0.030 & 0.038 & 0.046 & 0.029 & 0.020 & 0.033 \\
\hline$\Sigma \mathrm{REE}$ & 512 & 108 & 465 & 403 & 533 & 571 & 405 & 412 & 645 & 424 & 463 & 500 & 455 & 623 & 376 \\
\hline $\mathrm{La}_{\mathrm{N}} / \mathrm{Yb}_{\mathrm{N}}$ & 27.4 & 2.6 & 53.9 & 32.9 & 53.0 & 49.8 & 46.0 & 39.8 & 68.5 & 39.8 & 46.8 & 43.9 & 38.6 & 44.6 & 53.4 \\
\hline $\mathrm{Eu} / \mathrm{Eu}^{*}$ & 0.96 & 1.27 & 0.97 & 0.86 & 0.94 & 0.97 & 0.96 & 0.98 & 0.84 & 0.96 & 0.96 & 0.93 & 0.91 & 0.87 & 0.95 \\
\hline $\mathrm{Zr} / \mathrm{Hf}$ & 59.7 & 64.3 & 55.3 & 44.4 & 57.0 & 60.7 & 56.4 & 54.7 & 47.1 & 43.5 & 53.9 & 60.4 & 59.3 & 43.7 & 52.9 \\
\hline $\mathrm{Th} / \mathrm{U}$ & 2.47 & 0.10 & 4.46 & 4.10 & 4.02 & 3.58 & 4.78 & 3.75 & 5.27 & 3.11 & 3.89 & 3.50 & 4.48 & 3.46 & 4.02 \\
\hline $\mathrm{Nb} / \mathrm{Ta}$ & 25.5 & 303.1 & 20.5 & 16.2 & 21.8 & 26.7 & 21.0 & 22.7 & 16.7 & 51.8 & 24.8 & 21.6 & 23.4 & 22.1 & 18.6 \\
\hline $\mathrm{Nb} / \mathrm{U}$ & 39.2 & 24.5 & 56.6 & 50.2 & 48.7 & 39.9 & 55.4 & 50.9 & 39.6 & 48.8 & 51.3 & 46.2 & 40.7 & 31.8 & 51.7 \\
\hline $\mathrm{La} / \mathrm{Nb}$ & 0.64 & 0.34 & 0.69 & 0.67 & 0.82 & 0.86 & 0.77 & 0.68 & 1.01 & 0.53 & 0.69 & 0.82 & 0.78 & 0.88 & 0.71 \\
\hline $\mathrm{Ba} / \mathrm{Nb}$ & 5.13 & 17.65 & 6.18 & 6.03 & 4.12 & 5.59 & 5.48 & 6.40 & 3.63 & 7.72 & 6.48 & 5.22 & 6.04 & 11.8 & 5.77 \\
\hline
\end{tabular}


Tab. 2 Continued

Explanations page 56

\begin{tabular}{|c|c|c|c|c|c|c|c|c|c|c|c|c|c|c|c|}
\hline Locality & 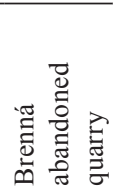 & 坣焉 & 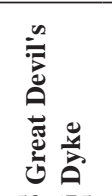 & 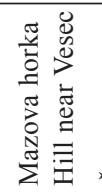 & 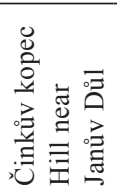 & 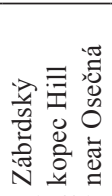 & 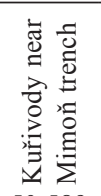 & 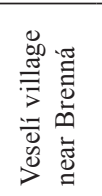 & 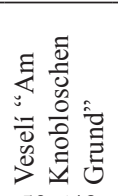 & 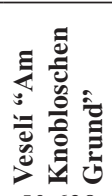 & 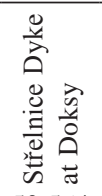 & 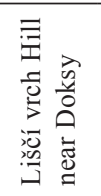 & 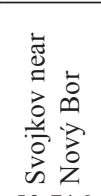 & 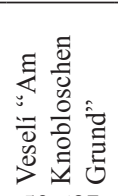 & 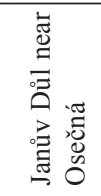 \\
\hline Latitute ${ }^{\circ} \mathrm{N}$ & 50.649 & 50.707 & 50.675 & 50.699 & 50.706 & 50.680 & 50.589 & 50.644 & 50.643 & 50.638 & 50.564 & 50.555 & 50.716 & 50.637 & 50.703 \\
\hline Longitude ${ }^{\circ} \mathrm{E}$ & 14.625 & 14.743 & 14.946 & 14.985 & 14.954 & 14.924 & 14.838 & 14.632 & 14.637 & 14.641 & 14.671 & 14.644 & 14.595 & 14.639 & 14.954 \\
\hline Sample & 67 & $\mathrm{P}-10^{*}$ & 30 & $\mathrm{P}-4 *$ & P-7* & P-8* & $\mathrm{P}-5^{*}$ & 49 & $49 \mathrm{~A}$ & $1 / 13$ & X-1 & 42 & 35 & $\mathrm{P}-1^{*}$ & P-3* \\
\hline Rock type & CPOL & CPOL & MON & MON & MON & MON & MON & MO & MO & MO & $\begin{array}{c}\mathrm{MO}- \\
\mathrm{CA}\end{array}$ & $\begin{array}{c}\mathrm{MO}- \\
\mathrm{CA}\end{array}$ & CA & CA & $\begin{array}{c}\text { CA-BA } \\
(?)\end{array}$ \\
\hline$\overline{\mathrm{SiO}_{2} \text { (wt. \%) }}$ & 36.01 & 33.75 & 37.98 & 39.48 & 38.86 & 39.28 & 35.86 & 40.92 & 39.81 & 38.94 & 39.90 & 42.30 & 47.17 & 47.78 & 40.57 \\
\hline $\mathrm{TiO}_{2}$ & 4.53 & 2.04 & 2.51 & 2.36 & 2.65 & 2.39 & 2.76 & 3.96 & 3.24 & 3.43 & 3.52 & 3.37 & 2.78 & 2.58 & 2.59 \\
\hline $\mathrm{Al}_{2} \mathrm{O}_{3}$ & 10.78 & 8.38 & 9.14 & 9.54 & 9.72 & 8.61 & 10.18 & 11.80 & 13.68 & 13.08 & 11.90 & 12.78 & 16.14 & 16.03 & 14.34 \\
\hline $\mathrm{Fe}_{2}^{2} \mathrm{O}_{3}$ & 10.14 & 5.57 & 4.11 & 4.94 & 6.48 & 6.53 & 7.49 & 2.91 & 6.83 & 6.62 & 5.62 & 6.20 & 3.99 & 4.79 & 6.57 \\
\hline $\mathrm{FeO}$ & 7.39 & 5.17 & 6.89 & 6.01 & 3.57 & 4.23 & 4.39 & 4.92 & 4.86 & 5.07 & 5.75 & 4.10 & 5.10 & 4.72 & 5.60 \\
\hline $\mathrm{MnO}$ & 0.22 & 0.20 & 0.18 & 0.18 & 0.18 & 0.20 & 0.21 & 0.08 & 0.19 & 0.16 & 0.17 & 0.15 & 0.15 & 0.16 & 0.22 \\
\hline $\mathrm{MgO}$ & 7.92 & 17.12 & 16.48 & 17.75 & 9.82 & 13.82 & 14.14 & 15.08 & 10.62 & 11.01 & 10.70 & 9.00 & 4.18 & 4.73 & 7.79 \\
\hline $\mathrm{aO}$ & 15.79 & 18.38 & 13.37 & 13.23 & 16.78 & 14.28 & 12.84 & 14.54 & 13.82 & 13.17 & 14.53 & 13.77 & 9.11 & 9.38 & 13.06 \\
\hline $\mathrm{Na}_{2} \mathrm{O}$ & 0.44 & 1.62 & 3.05 & 2.48 & 1.26 & 1.60 & 0.49 & 1.57 & 0.80 & 0.89 & 1.37 & 1.84 & 4.01 & 2.82 & 1.36 \\
\hline $\mathrm{K}_{2} \mathrm{O}^{-}$ & 1.37 & 1.48 & 1.80 & 1.43 & 0.65 & 1.52 & 0.87 & 2.40 & 0.84 & 1.90 & 1.63 & 1.71 & 3.63 & 3.34 & 1.63 \\
\hline $\mathrm{P}_{2}^{2} \mathrm{O}_{5}$ & 1.35 & 1.31 & 0.93 & 0.69 & 0.81 & 1.00 & 0.91 & 0.35 & 0.52 & 0.53 & 0.55 & 0.60 & 0.52 & 0.50 & 1.04 \\
\hline $\mathrm{H}_{2} \mathrm{O}^{+}$ & 3.12 & 3.68 & 2.04 & 0.89 & 4.21 & 3.17 & 6.29 & 1.38 & 2.93 & 3.38 & 2.80 & 1.82 & 2.21 & 2.38 & 3.54 \\
\hline $\mathrm{H}_{2}^{-} \mathrm{O}^{-}$ & 0.45 & 0.22 & 0.33 & 0.12 & 1.30 & 0.60 & 1.30 & 0.06 & 0.84 & 1.48 & 0.78 & 1.06 & 0.57 & 0.16 & 0.66 \\
\hline $\mathrm{CO}_{2}$ & 0.27 & 0.42 & 0.56 & 0.53 & 3.15 & 2.32 & 1.63 & 0.20 & 0.53 & 0.32 & 0.31 & 1.03 & 0.52 & 0.09 & 0.51 \\
\hline $\mathrm{F}_{2}$ & 0.14 & n.d. & 0.12 & n.d. & n.d. & n.d. & n.d. & 0.25 & n.d. & n.d. & 0.03 & 0.28 & 0.19 & n.d. & n.d. \\
\hline $\mathrm{S}^{2}$ & n.d & n.d. & 0.04 & n.d. & n.d. & n.d. & n.d. & n.d & n.d. & n.d. & 0.05 & 0.07 & 0.03 & n.d. & n.d. \\
\hline Total & 99.92 & 99.58 & 99.53 & 99.73 & 99.59 & 99.70 & 99.57 & 100.42 & 99.51 & 99.98 & 99.61 & 100.08 & 100.30 & 99.58 & 99.62 \\
\hline $\mathrm{Ni}$ (ppm) & 7 & 310 & 575 & 400 & 160 & 262 & 244 & 85 & 72 & 134 & 78 & 111 & 23 & 27 & 49 \\
\hline $\mathrm{Cr}$ & 8 & 646 & 1190 & 984 & 595 & 582 & 706 & 242 & 175 & 262 & 184 & 172 & 65 & 75 & 114 \\
\hline Co & 43.1 & 58.0 & 65.0 & 68.0 & 49.0 & 52.0 & 59.0 & 48.1 & 43.6 & 50.5 & 44.5 & 48.1 & 30.4 & 30.0 & 41.0 \\
\hline $\mathrm{Sc}$ & 23.6 & 25.1 & 35.8 & 28.2 & 29.0 & 25.5 & 30.7 & 52.0 & 44.4 & 45.4 & 42.5 & 50.2 & 25.4 & 19.6 & 22.8 \\
\hline $\mathrm{Rb}$ & 49.4 & 46.0 & 55.1 & 47.0 & 211 & 126 & 47.0 & 133 & 97.5 & 154 & 102 & 123 & 121 & 91.0 & 101 \\
\hline $\mathrm{Sr}$ & 2123 & 1801 & 1005 & 813 & 1130 & 1135 & 1621 & 725 & 1017 & 854 & 1020 & 816 & 835 & 894 & 1045 \\
\hline $\mathrm{Y}$ & 29 & 33 & 17 & 20 & 26 & 33 & 31 & 21 & 21 & 21 & 20 & 23 & 23 & 25 & 30 \\
\hline $\mathrm{Zr}$ & 491 & 295 & 253 & 233 & 2. & 48 & 330 & 278 & 278 & 301 & 269 & 291 & 354 & 308 & 272 \\
\hline $\mathrm{Nb}$ & 120 & 173 & 121 & 98 & 115 & 143 & 156 & 69 & 66.5 & 78.7 & 67.2 & 70.1 & 79.8 & 86.0 & 103 \\
\hline Cs & 0.8 & 0.63 & 0.90 & 0.59 & 1.75 & 1.99 & 1.64 & 6.14 & 2.53 & 1.89 & 2.99 & 1.82 & 1.98 & 1.11 & 1.57 \\
\hline $\mathrm{Ba}$ & 540 & 1229 & 988 & 687 & 949 & 1162 & 1900 & 936 & 560 & 1606 & 688 & 834 & 649 & 1146 & 886 \\
\hline $\mathrm{La}$ & 84.7 & 126 & 51.2 & 52.1 & 79.7 & 97.7 & 103 & 47.6 & 49.3 & 51.3 & 47.2 & 53.5 & 60.2 & 61.0 & 68.7 \\
\hline Ce & 175 & 249 & 97.0 & 99.5 & 151 & 203 & 202 & 98.0 & 102 & 104 & 99.8 & 108 & 115 & 118 & 137 \\
\hline Pr & & .5 & 1 & 11.6 & & 5 & 3 & 11. & 1 & 2.5 & 11.9 & 12.7 & .7 & 3.1 & 15.5 \\
\hline $\mathrm{Nd}$ & 2.6 & 91.6 & 45.9 & 44.9 & 61.4 & .7 & 77.0 & 47.2 & 49.4 & 50.6 & 48.2 & 51.7 & 49.1 & 48.2 & 59.0 \\
\hline $\mathrm{Sm}$ & 1 & 15.6 & 8 & 8.70 & 10.9 & 14.2 & 13.2 & 8. & 9. & 9.27 & 8.87 & 9.40 & 8.5 & 8.60 & 10.6 \\
\hline Eu & 4.23 & 4.40 & 2.41 & 2.57 & 3.33 & 4.17 & 3.93 & 2.80 & 2.74 & 2.74 & 2.64 & 2.98 & 2.68 & 2.50 & 3.15 \\
\hline $\mathrm{Gd}$ & 12.7 & 16.2 & & 8.33 & & 14.7 & 14.0 & 7.80 & 8.09 & 8.61 & 7.96 & 8.56 & 7.91 & 8.92 & 10.9 \\
\hline $\mathrm{Tb}$ & & & & 1.02 & & & 160 & & 1.08 & & 0 & 11 & 1 & 1.07 & 1.34 \\
\hline$y$ & & & & 4.29 & & & 6.27 & & 5. & 4. & 4.91 & 2 & 5 & 4.74 & 5.83 \\
\hline Но & 1.36 & 1.13 & 0.72 & 0 & 0.92 & 1.1 & 1. & 0. & 0.93 & 0. & 0.88 & 0.96 & 0.98 & 0.84 & 1.04 \\
\hline Er & 3.3 & 3.06 & 1.95 & 1.8 & & 3.11 & 2.91 & 2. & 2.30 & 2. & 2.18 & 2.38 & 2. & 2.49 & 3.09 \\
\hline $\mathrm{Tm}$ & 0.41 & 0.32 & 0.22 & 0.20 & 0.28 & 0.32 & 0.29 & 0.24 & 0.28 & 0.26 & 0.28 & 0.29 & 0.33 & 0.29 & 0.35 \\
\hline $\mathrm{Yb}$ & 219 & 1.97 & 1.39 & 1.31 & 171 & 2.08 & 1.94 & & 1.63 & 1.92 & 9 & 1.65 & 1.92 & 2.06 & 2.41 \\
\hline $\mathrm{Lu}$ & & & & 0 . & & & & & 0.23 & 0. & 2 & 0.22 & 0. & 0.28 & 0.31 \\
\hline $\mathrm{Hf}$ & 10. & & & & & & & & 6. & 10 & , & 5 & 6 & 7.29 & 6.56 \\
\hline Та & & & 6 & 5. & 6 & 7.89 & & 3. & 3. & 3. & 3.98 & 3.67 & 3.78 & 4.65 & 6.16 \\
\hline Th & 7.94 & 13.9 & 6.87 & 5.99 & 9.83 & 10.6 & 13.1 & 4.84 & 6.89 & 12.3 & 7.98 & 5.59 & 8.75 & 10.0 & 7.33 \\
\hline 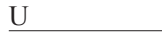 & 2.12 & 3.76 & 1.89 & 1.62 & 2.40 & 3.02 & 3.74 & 1.20 & 1.85 & 1.45 & 1.92 & 1.44 & 2.20 & 2.48 & 1.84 \\
\hline $\mathrm{Mg} \#$ & 50.2 & 77.9 & 76.6 & 78.1 & 68.7 & 74.2 & 72.7 & 80.8 & 66.9 & 67.7 & 67.5 & 66.1 & 50.2 & 52.4 & 58.7 \\
\hline$/ \mathrm{Rb}$ & 230 & 267 & 271 & 253 & 26 & 100 & 154 & 150 & 72 & 102 & 133 & 115 & 249 & 305 & 134 \\
\hline $\mathrm{b} / \mathrm{S}$ & 0.023 & 0.026 & 0.055 & 0.058 & 0.187 & 0.111 & 0.029 & 0.183 & 0.096 & 0.180 & 0.100 & 0.151 & 0.145 & 0.102 & 0.097 \\
\hline REE & 412 & 544 & 235 & 237 & 346 & 451 & 449 & 235 & 245 & 250 & 238 & 259 & 269 & 272 & 319 \\
\hline $\mathrm{La}_{\mathrm{N}} / \mathrm{Yb}$ & 27.7 & 45.9 & 26.4 & 28.5 & 33.4 & 33.7 & 38.1 & 23.7 & 21.7 & 19.2 & 21.3 & 23.3 & 22.5 & 21.2 & 20.4 \\
\hline $\mathrm{Eu} / \mathrm{Eu}^{*}$ & 0.94 & 0.84 & 0.86 & 0.91 & 0.92 & 0.87 & 0.88 & 1.03 & 0.96 & 0.92 & 0.94 & 1.00 & 0.98 & 0.87 & 0.89 \\
\hline $\mathrm{Zr} / \mathrm{Hf}$ & 47.9 & 47.1 & 43.0 & 40.7 & 41.7 & 44.8 & 43.0 & 45.8 & 41.6 & 29.5 & 38.3 & 45.1 & 53.0 & 42.2 & 41.5 \\
\hline $\mathrm{Th} / \mathrm{U}$ & 3.75 & 3.70 & 3.63 & 3.70 & 4.10 & 3.51 & 3.50 & 4.03 & 3.72 & 8.45 & 4.16 & 3.88 & 3.98 & 4.03 & 3.98 \\
\hline$J_{1}$ & & 20.9 & 19.2 & 16.8 & 18 & 18.1 & 19.3 & 20.1 & 7.9 & 22.6 & 16.9 & 19. & 21.1 & 18.5 & 16.7 \\
\hline $\mathrm{D} / \mathrm{U}$ & & 46.0 & 64.0 & 60.5 & 4.9 & 47.4 & 41.7 & 57. & 35.9 & 54.3 & 35.0 & 48.7 & 36.3 & 34.7 & 56.0 \\
\hline $\mathrm{La} / \mathrm{Nb}$ & 0.71 & 0.73 & & 0.53 & 0.69 & 0.68 & 0.66 & 0.69 & 0.74 & 0.65 & 0.70 & 0.76 & 0.75 & 0.71 & 0.67 \\
\hline $\mathrm{Ba} / \mathrm{Nb}$ & 4.50 & 7.10 & 8.17 & 7.01 & 8.25 & 8.13 & 12.2 & 13.5 & 8.42 & 20.4 & 10.2 & 11.9 & 8.13 & 13.3 & 8.60 \\
\hline
\end{tabular}




\section{Explanations for Tab. 2:}

type localities of Scheumann (1913) in bold

OM - olivine melilitolite, PHL/PEG - phlogopitite - glimmerite in OM pegmatoid, OMM - olivine micro-melilitolite "chilled margin" of OM, POL-OMM - polzenite - olivine micro-melilitolite (dykes of the vesecite type - Scheumann 1913), POL - polzenite (dykes of the modlibovite type - Scheumann 1913),

CPOL - clinopyroxene "polzenite" (dykes of the luhite type - Scheumann 1913), MON - melilite-bearing olivine nephelinite, MO - monchiquite ("wesselite"), CA - camptonite ("wesselite"), MO-CA - monchiquite-camptonite, CA-BA (?) - camptonite-basanite (?)

$\mathrm{Mg} \#=100 \mathrm{Mg} /\left(\mathrm{Mg}+\mathrm{Fe}^{2+}\right)$, for $\mathrm{Fe}^{3+} / \mathrm{Fe}=0.15$; n.d. - not determined, unpubl - unpublished

Analysts: wet analyses by P. Povondra and V. Vonásková (analyses with an asterisk) both from Faculty of Science, Charles University in Prague; trace-element analyses by ICP MS [J. Durišová, Institute of Geology, Acad Sci. CR and L. Strnad, Faculty of Science, Charles University in Prague (analyses with an asterisk)]

Tab. 3 Comparison of geological, mineralogical, petrologic and geochemical characteristics of the melilitic and monchiquite-camptonite rock series of the Ploučnice River Region

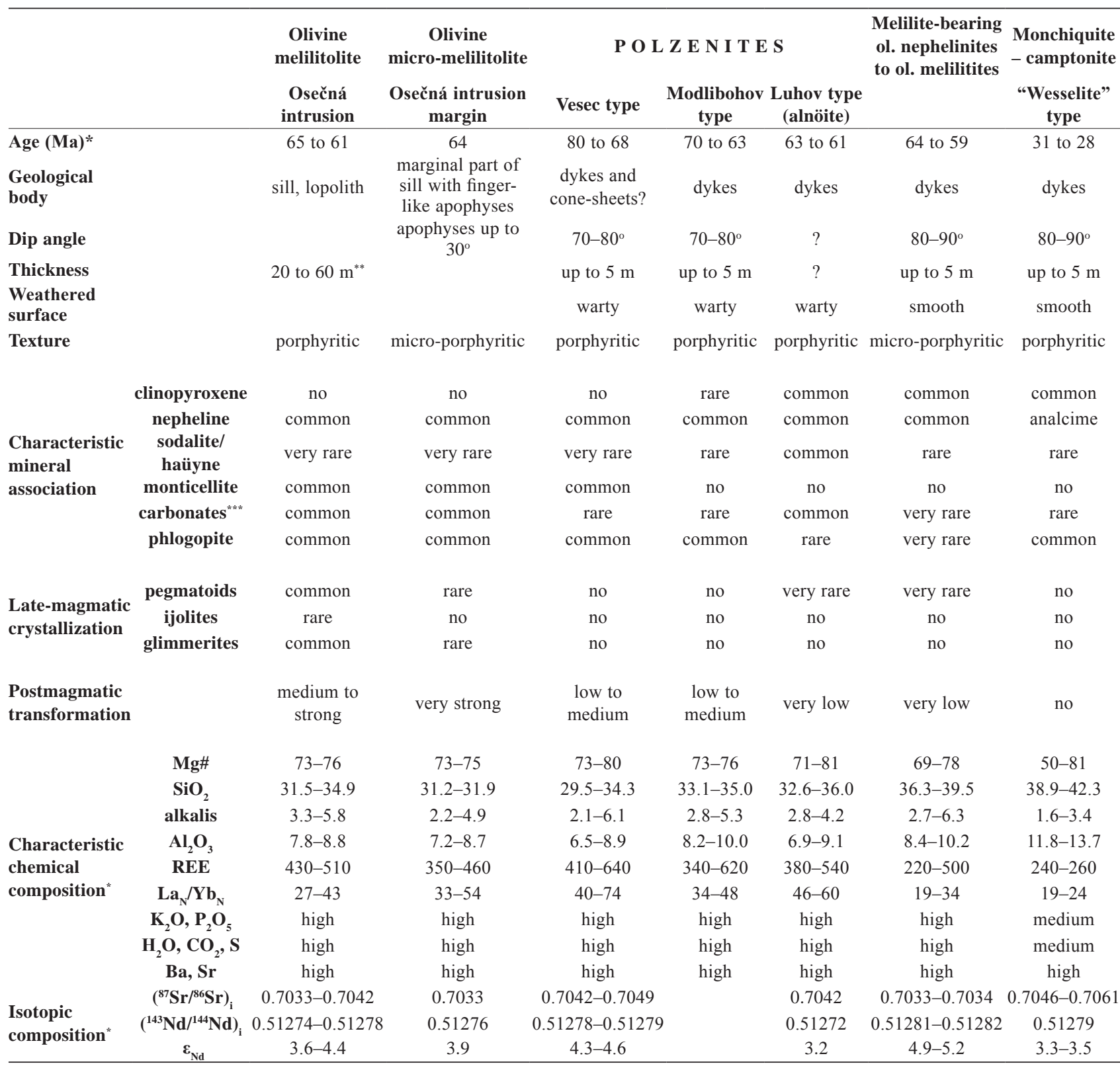

" geochemical data from this publication and Ulrych et al. (2008)

** apparent thickness from boreholes

**** secondary $>$ primary

$\mathrm{Mg} \#=100 \mathrm{Mg} /\left(\mathrm{Mg}+\mathrm{Fe}^{2+}\right)$, for $\mathrm{Fe}^{3+} / \mathrm{Fe}=0.15$ 


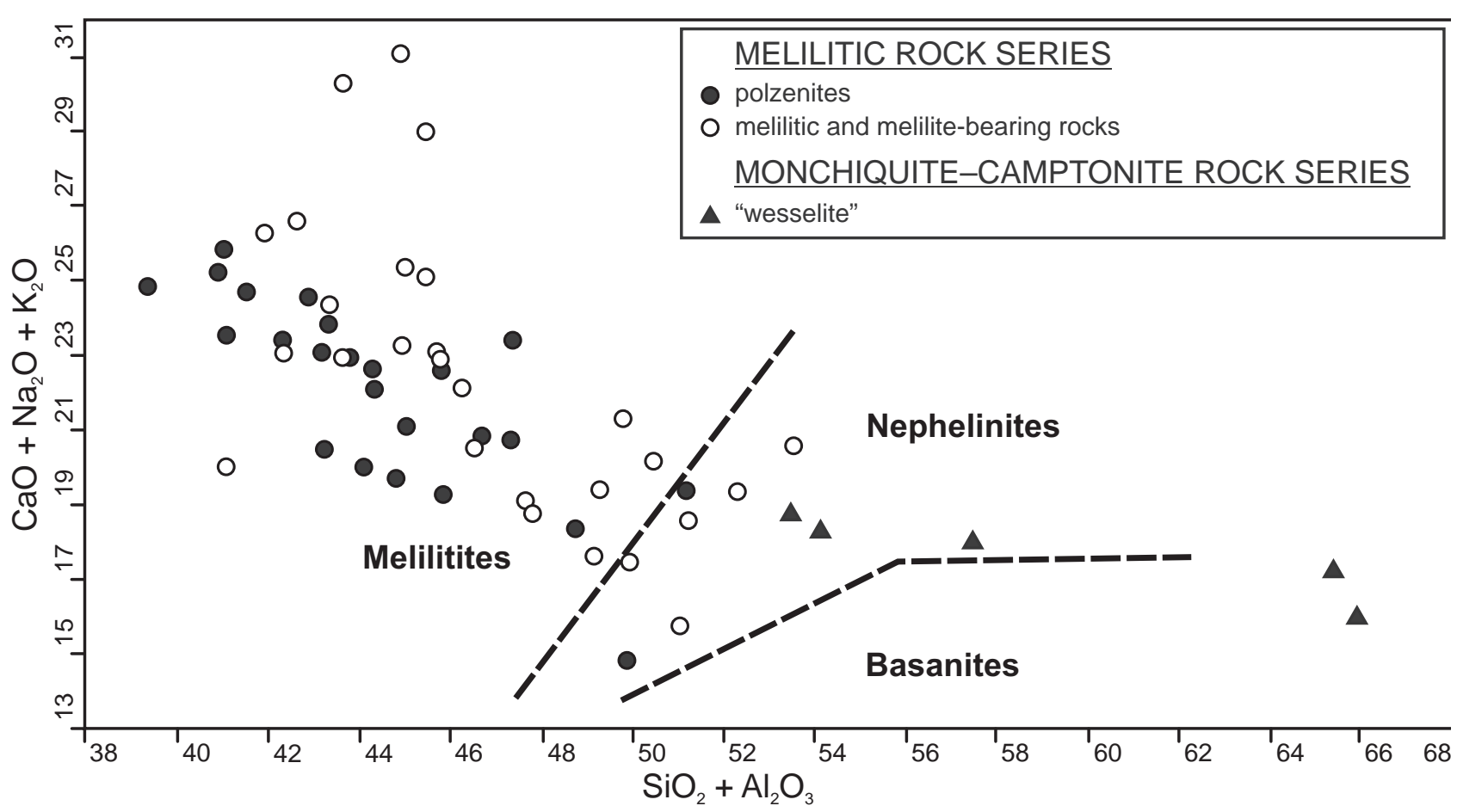

Fig. 4 A binary $\mathrm{CaO}+\mathrm{Na}_{2} \mathrm{O}+\mathrm{K}_{2} \mathrm{O}$ vs. $\mathrm{SiO}_{2}+\mathrm{Al}_{2} \mathrm{O}_{3}$ (wt. \%) diagram for the melilitic rock series and monchiquite-camptonite rock series (Le Bas 1989). Data from Ulrych et al. (2008) and this work.

of late-magmatic and post-magmatic enrichments have been recognized:

Ca-Na - $\left(\mathrm{H}_{2} \mathrm{O}, \mathrm{P}, \mathrm{F}\right)$ which produced melilitolite pegmatoids containing melilite + nepheline + fluorapatite, $(\mathrm{F}, \mathrm{OH})$-bearing titanian andradite, fluorite, wollastonite and thomsonite.

$\mathrm{Na}-\left(\mathrm{CO}_{2}, \mathrm{Zr}\right)$ which mainly led to the production of ijolites (nepheline $+\mathrm{Na}$-rich diopside + calcite, calzirtite and pectolite), see a similar paragenesis of pegmatoids in olivine nephelinites from western Bohemia (Ulrych et al. 2000a, 2005)

$\mathbf{K}-\left(\mathrm{Fe}^{3+}, \mathrm{Ti}, \mathrm{Zr}, \mathrm{F}, \mathrm{S}\right)$ resulting in the formation of glimmerites formed by (Ti,Ba)-rich phlogopite + (Ti,Zr)andradite, pyrite and rasvumite.

Nevertheless, the process of metasomatic transformation of melilitic rocks was complex. It included monticellitization, glimmeritization, garnetization and transformation of groundmass to a mixture of carbonates, zeolites, and pectolite (cebollitization). The alterations concentrated to intensively water-saturated sheets of olivine micro-melilitolite in Cretaceous sediments.

Geochemical data on the melilitolite pegmatoids and ijolite dykes presented by Ulrych et al. (2008) display lower contents of transition trace elements such as $\mathrm{Cr}, \mathrm{Ni}$ and Co compared to the host olivine melilitolite (Tab. 2) and glimmerites. On the other hand, the concentrations of $\mathrm{P}, \mathrm{Sr}, \mathrm{Ba}, \mathrm{Y}, \mathrm{REE}, \mathrm{U}, \mathrm{Zr}$ and $\mathrm{Nb}$ are slightly elevated.

Primitive mantle-normalized multielement plots for melilitic rocks show enrichment in incompatible ele- ments (Fig. 5). Melilite-bearing olivine nephelinites of the Devil's Walls dyke swarm have similar geochemical characteristics as those of olivine micro-melilitolites and polzenites of the Osečná Complex, although the concentrations of incompatible elements in the Devil's Walls dykes tend to be lower. The pronounced negative $\mathrm{K}$ anomaly in all samples is most characteristic.

Melilitic rocks are enriched in rare earth elements ( 2 REE 220-640 ppm) compared to primitive upper mantle (Sun and McDonough 1989) (Tab. 2, Fig. 6). They are similarly enriched in LREE relative to HREE with high $\mathrm{La}_{\mathrm{N}} / \mathrm{Yb}_{\mathrm{N}}$ ratios. Ultramafic lamprophyres, olivine melilitolites and micro-melilitolites have mostly higher $\mathrm{La}_{\mathrm{N}} / \mathrm{Yb}_{\mathrm{N}}$ ratios $(27-68)$ than melilite-bearing olivine nephelinites $\mathrm{La}_{\mathrm{N}} / \mathrm{Yb}_{\mathrm{N}}(20-38)$ although the differences among the bulk-rock compositions are subtle. No samples exhibit substantial negative Eu anomalies $\left(\mathrm{Eu} / \mathrm{Eu}^{*}=\right.$ 0.84-0.98).

\subsection{Alkaline lamprophyres}

Chemical compositions of "wesselite" (monchiquite to camptonite) dykes $(\mathrm{Mg} \#=50-68$ but also as high as 81 !) (Scheumann 1913, 1922; Ulrych et al. 1990, 1998; Kühn 1999) from the PRR display partly variable chemical characteristics (see Tab. 2) and plot prevalently to basanite in the TAS diagram (Fig. 3). The monchiquite from Veselí (samples 49A and 1/13) shows medium Mg\# values of 67-68. Camptonites from the Střelnice dyke at Doksy 


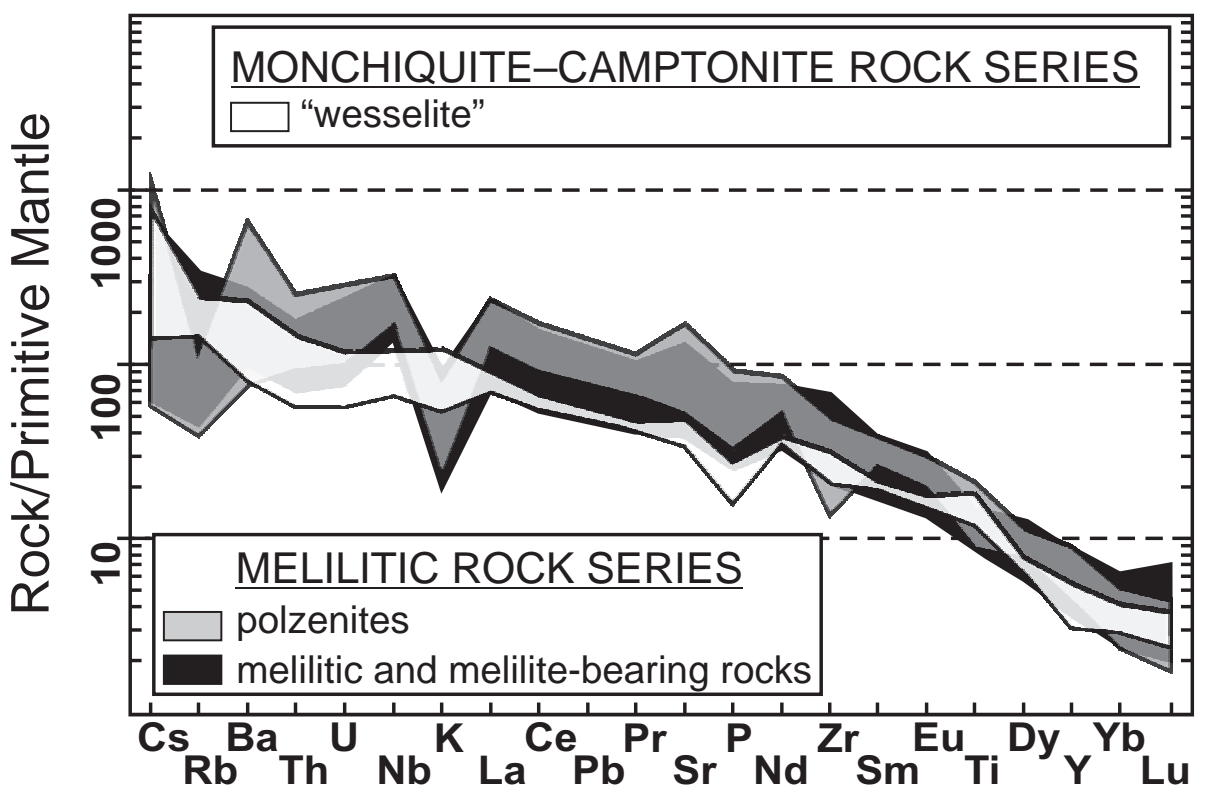

Fig. 5 Primitive mantle-normalized (Sun and McDonough 1989) trace-element patterns for volcanic rocks of the Ploučnice River Region. Shaded fields represent the compositional ranges of the melilitic rock series (lamprophyres - polzenites and other melilitic and melilite-bearing rocks) and the monchiquite-camptonite rock series ("wesselites"). Data from Ulrych et al. (2008) and this work.
(X-1) and Liščí vrch Hill near Doksy (sample 42) are characterized by similar Mg\# (66-67). Samples from Veselí P-1* (Fig. 2e) and Svojkov (sample 35) are leucocratic and display very low Mg\# of 50-52, while that from Veselí (sample 49) is melanocratic with very high $\mathrm{Mg \#} \mathrm{(81).}$

Primitive mantle-normalized multielement plots for alkaline lamprophyres of the "wesselite" type show a degree of enrichment in incompatible elements similar to that in the compared melilitic rocks (Fig. 5) but lack a negative $\mathrm{K}$ anomaly.

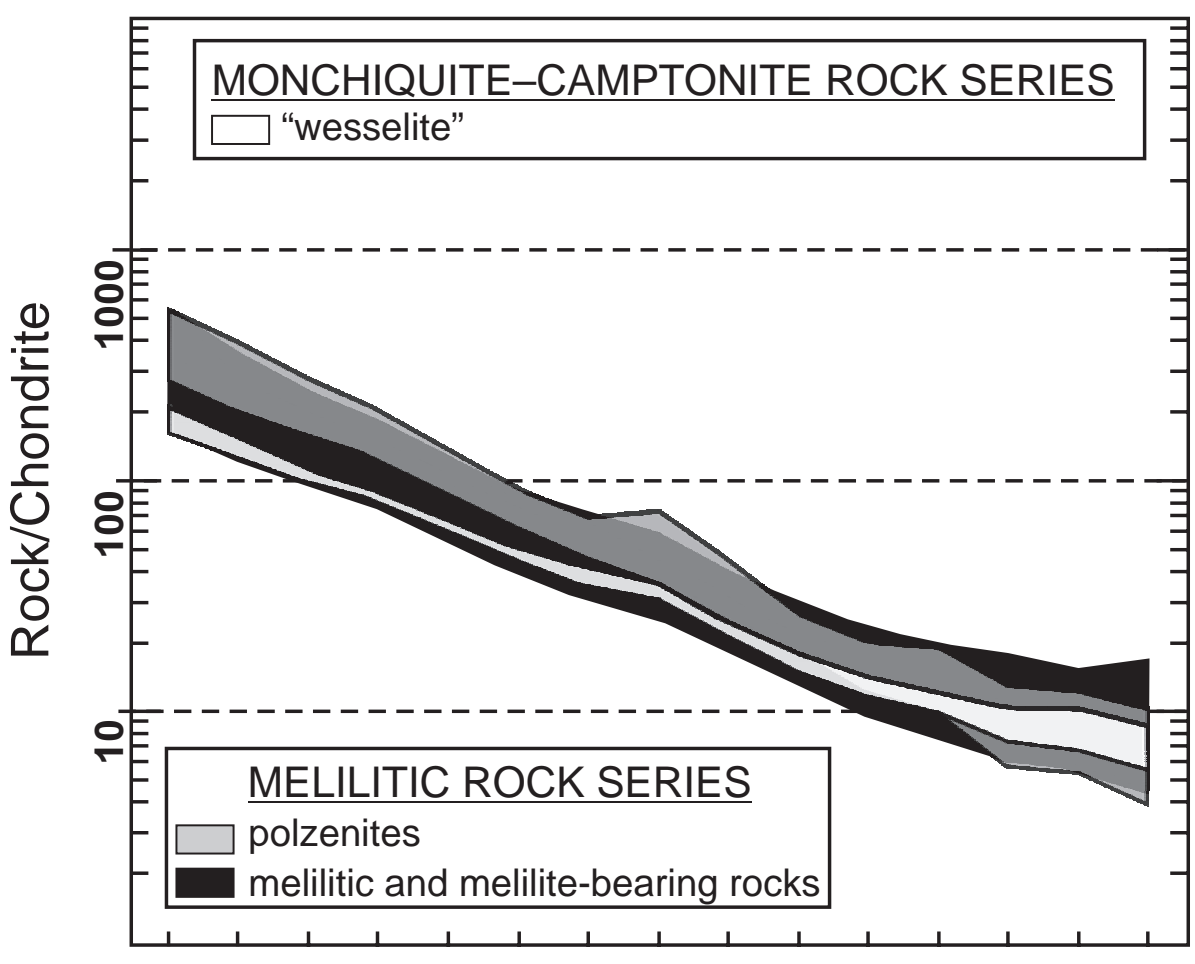

La Ce Pr Nd PmSmEu Gd Tb Dy Ho Er Tm Yb Lu
The alkaline lamprophyres are moderately enriched in LREE relative to HREE $\left(\mathrm{La}_{\mathrm{N}} / \mathrm{Yb}_{\mathrm{N}}=20-24\right.$ : Tab. 2, Fig. 6). On average, the enrichment in LREE abundances of these rocks is lower than that of the compared melilitic rocks.

\section{3. $K-A r$ age determinations}

New K-Ar age determinations (80-61 Ma, for errors see Tab. 4) of the polzenite types of vesecite (80-68 Ma), modlibovite (70-63 Ma) and luhite (63-61 Ma) from the original localities (Tab. 4) expand the hitherto presented Late Cretaceous to Palaeocene age span for the melilitic volcanic rock series of the PRR (68-59 Ma; Ulrych et al. 2008).

However, $\mathrm{K}-\mathrm{Ar}$ data for original "wesselite" (monchiquite) dyke sample 1/13 are 30.9 $\mathrm{Ma}$ (phlogopite) to $27.8 \mathrm{Ma}$ (kaersutite), while the age of the "wesselite" (camptonite) dyke sample P-1* (whole-rock data) is $23.2 \mathrm{Ma}$, see Tab. 4.

Fig. 6 Chondrite-normalized (Boynton 1984) REE patterns for the studied volcanic rocks. Shaded fields represent the compositional ranges of the melilitic rock series (lamprophyres - polzenites and other melilitic and melilite-bearing rocks) and the monchiquite-camptonite rock series ("wesselites"). Data from Ulrych et al. (2008) and this work. 
Tab. $4 \mathrm{~K}-\mathrm{Ar}$ ages for the studied volcanic rocks

\begin{tabular}{|c|c|c|c|c|c|c|}
\hline Sample & Locality & Rock type & $\mathrm{K}(\mathrm{wt} . \%)$ & $\begin{array}{c}{ }^{40} \mathrm{Ar}(\mathrm{rad}) 10^{-6} \\
\mathrm{ccSTP} / \mathrm{g}\end{array}$ & $\begin{array}{c}{ }^{40} \mathrm{Ar}(\mathrm{rad}) \\
(\%)\end{array}$ & $\begin{array}{c}\text { Age } \pm 1 \sigma \\
(\mathrm{Ma})\end{array}$ \\
\hline \multicolumn{7}{|c|}{ Melilitic rock series } \\
\hline POL-57 & Děvín Hill near Hamr & Polzenite - vesecite & 0.971 & $3.067 \times 10^{-6}$ & 47.7 & $79.5 \pm 3.5$ \\
\hline$P *-2$ & Modlibohov & Polzenite - modlibovite & 1.318 & $3.636 \times 10^{-6}$ & 40.9 & $69.6 \pm 3.0$ \\
\hline$P *-10$ & Luhov & Clinopyroxene "polzenite" - luhite (ailikite) & 1.102 & $2.672 \times 10^{-6}$ & 43.9 & $61.3 \pm 2.6$ \\
\hline $\mathrm{P}^{*}-4$ & Mazova horka Hill near Vesec & melilite-bearing olivine nephelinite & 1.142 & $2.795 \times 10^{-6}$ & 60.7 & $61.9 \pm 2.4$ \\
\hline \multicolumn{7}{|c|}{ Monchiquite-camptonite rock series } \\
\hline 1/13-1 & Veselí, “Am Knobloschen Grund” & phlogopite in monchiquite - "wesselite" & 7.617 & $9.234 \times 10^{-6}$ & 79.5 & $30.9 \pm 1.2$ \\
\hline $1 / 13-2$ & Veselí, “Am Knobloschen Grund” & kaersutite in monchiquite - "wesselite" & 1.569 & $1.711 \times 10^{-6}$ & 74.6 & $27.8 \pm 1.1$ \\
\hline $\mathrm{P}^{*}-1$ & Veselí, “Am Knobloschen Grund” & camptonite - "wesselite" & 2.993 & $2.713 \times 10^{-6}$ & 45.3 & $23.2 \pm 1.0$ \\
\hline $\mathrm{P} *-3 \mathrm{~A}$ & Janův Důl near Osečná & matrix of camptonite - basanite (?) & 1.649 & $1.494 \times 10^{-6}$ & 26.5 & $23.1 \pm 1.2$ \\
\hline $\mathrm{P}^{*}-3 \mathrm{~B}$ & Janův Důl near Osečná & kaersutite in camptonite - basanite (?) & 1.246 & $1.401 \times 10^{-6}$ & 22.9 & $28.7 \pm 1.6$ \\
\hline
\end{tabular}

Vesecite type locality - Děvín Hill, modlibovite type locality - Modlibohov, luhite type locality - Luhov; type localities of Scheumann (1913) in bold

The results correspond well with new $\mathrm{K}-\mathrm{Ar}$ data for the camptonite-basanite (?) dyke from Janův Důl locality, sample $\mathrm{P}^{*}-3$ : $28.7 \mathrm{Ma}$ for kaersutite phenocrysts and 23.1 Ma for groundmass (Tab. 4).

\section{Discussion}

\subsection{Problems of the polzenite and associated rock classification}

Regarding the classification of polzenites, there are currently two equivalent approaches. According to the first view, polzenites represent only lamprophyric, i.e. volatile-rich, facies ("lamprophyre clan") of melilite-bearing group of rocks sensu Mitchell (1994) (see Woolley et al. 1996; Le Maitre 2002). This is based on the assumption that the term lamprophyre has no genetic significance and the recognition of a lamprophyre facies is proposed as a means of conveying the concept that some members of a cogenetic petrological series crystallized under volatilerich conditions (Mitchell 1994). The textural, modal and chemical similarity of polzenites (in particular of the Vesec type) and marginal olivine micro-melilitolite facies of the Osečná intrusion support this view. Nevertheless, the actual Osečná olivine melilitolite sheet can represent a body of lamprophyre or a lamprophyric facies of olivine melilitolite.

According to the second view, polzenites belong to a separate group of ultramafic lamprophyres (Rock 1987, 1991; Le Maitre 1989) as they meet all criteria required by Le Maitre (1989) and Rock (1991) for lamprophyres. However, the polzenites were considered a (more felsic) variant of alnöite by some authors (Tappe et al. 2005). The original Scheumann's (1913) definition based, contrary to alnöite, on clinopyroxene-free mineral composition was not taken in consideration. Tappe at al. (2005) introduced a critical modification to the Le Maitre (2002) classification, reintroducing the ultramafic lamprophyres as inequigranular textured rocks with olivine and phlogopite macrocrysts and/or phenocrysts. Nevertheless, they recognized only three end-members: alnöite (essential groundmass phase melilite), aillikite (essential groundmass primary carbonate) and damtjernite (essential groundmass nepheline and/or alkali feldspar) (Tab. 1). With respect to the petrography of clinopyroxene-free polzenite (the Vesec type; typical $\mathrm{Mg} \#=76-80$ ), this polzenite can be considered a valid end-member of the ultramafic lamprophyre group in accord with original Scheumann's (1913) definition (Tab. 1).

The similarity of polzenite dykes of the Vesec type and the olivine micro-melilitolite of the Osečná intrusion in porphyritic texture, modal and chemical compositions is striking (see Tab. 3). Notable is also the considerably close mean composition of the volatile-rich porphyritic medium-grained olivine melilitolite of the Osečná intrusion with high phlogopite content ( 14 vol. \% - Pivec et al. 1998) and polzenites of the Vesec type and olivine micro-melilitolites with only half amount of poikilitic phlogopite ( 7 vol. \% - Pivec et al. 1998).

Pivec et al. (1986) and Ulrych et al. (1988) considered the Modlibohov type a typical polzenite despite minor clinopyroxene contents (up to 2.5 vol. \%). The term "clinopyroxene polzenite" for the Luhov type (clinopyroxene up to 20 vol. \%) of Scheumann (1922) is not correct as its modal composition corresponds to alnöite sensu Rosenbusch (1887). Scheumann (1913) saw the fundamental differences between polzenite and alnöite in the absence of clinopyroxene and the presence of haüyne in the former rock type. However, only samples of the Vesec type contain no clinopyroxene. The modal composition testifies for a continuously increasing presence of clinopyroxene at the expense of melilite and partly also phlogopite in the modlibovite-luhite series. The Luhov 
type alnöites are characterized by higher $\mathrm{SiO}_{2}$ contents (by $\sim 2$ wt. \%) and lower $\mathrm{MgO}$ and $\mathrm{CaO}$ contents (by $\sim 2$ wt. \%) compared to the pyroxene-free Vesec type.

The present classifications, including that of Le Maitre (1989) and Rock (1991), do not consider these characteristics (especially the absence of clinopyroxene!) at all. The presence of clinopyroxene and Na-bearing foids is not considered diagnostic for the discrimination between polzenite and alnöite in these classifications, and nepheline is not considered typical of polzenite. Nevertheless, nepheline is the most characteristic mineral of the studied polzenites (Scheumann 1913, 1922; Ulrych et al. 1991; Pivec et al. 1998). The presence of magmatic carbonate is characteristic of alnöite but minor primary carbonate should be present in polzenites as well.

Vesecite is the oldest and most characteristic polzenite type in the PRR. Pfeiffer (1994) published a K-Ar wholerock age of 71.3 Ma for the famous Zeughausgang olivine melilitite (polzenite) dyke in Saxony. Alnöite of the Delitzsch Complex yielded a $\mathrm{Rb}-\mathrm{Sr}$ phlogopite age of $73 \pm 2 \mathrm{Ma}$ (Krüger et al. 2013). Ultramafic lamprophyre from Ebersbach, Lusatia shows substantially higher ages (K-Ar on phlogopite: $130 \pm 5 \mathrm{Ma}-$ Renno et al. 2003a and $\mathrm{Ar}-\mathrm{Ar}$ on phlogopite: $126.64 \pm 0.27 \mathrm{Ma}-$ Renno et al. 2003b). The new K-Ar dating of "wesselite" samples confirms an affinity of the monchiquite-camptonite series to Oligocene volcanic rocks of the tephrite/basanite-phonolite/trachyte rock series (42 to $16 \mathrm{Ma}$ ) of the syn-rift period of the northwestern Bohemian Massif (Ulrych et al. 2011).

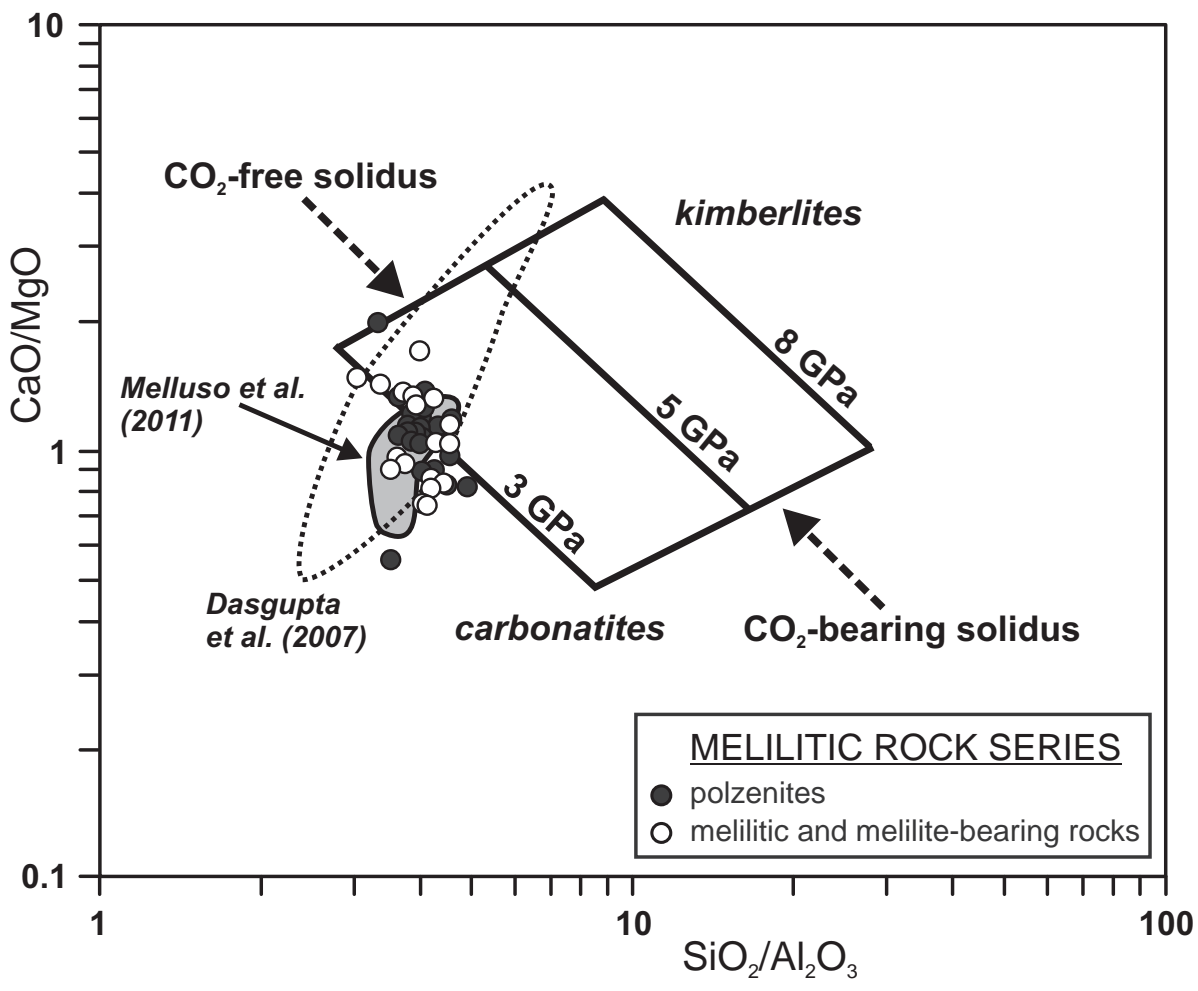

\subsection{Nature, source and evolution of magma}

Geological and geochemical characteristics of melilitic and melilite-bearing rocks of the PRR point to a single rock series (Tab. 3). The limited volume and high $\mathrm{Mg} \#$ (73-81) of this melilitic rock series suggest an origin by low degrees of fertile (carbonate-enriched) lithospheric mantle melting with residual garnet in a peridotitic source. This is consistent with high $\mathrm{La}_{\mathrm{N}} / \mathrm{Yb}_{\mathrm{N}}$ values (Tab. 3). Some of the samples reach the Mg\# up to 80-81 characteristic of mafic cumulates (Rhodes 1981).

A specific type of olivine-free, low-Mg\# (50), $\mathrm{Fe}_{2} \mathrm{O}_{3}$ (10.1 wt. \%) and $\mathrm{TiO}_{2}$-rich (4.5 wt. \%) clinopyroxenebearing (alnöitic) rock comes from Brenná (sample 67). More evolved melilitites described from Tanzania with lower \#Mg ( 60), low $\mathrm{Ni}(\sim 100 \mathrm{ppm})$, but rich in $\mathrm{Ti}, \mathrm{Ca}$, $\mathrm{Na}, \mathrm{K}$, and poor in $\mathrm{Al}$ are interpreted as the result of olivine fractionation of parental nephelinite melt (Dawson et al. 1985).

In contrast, the younger monchiquite-camptonite dyke series is characterized by substantially lower $\mathrm{Mg} \#$ (50-67) and lower contents of compatible elements (in particular $\mathrm{Ni}$ and $\mathrm{Cr}$ ) compared to those of the melilitic rock series. They are comparable with the Oligocene basanite lava flows of the České středohoří Mts. $(\mathrm{Mg} \#=$ 41-58) that originated in Ohře/Eger continental rift setting (Ulrych et al. 2002).

Wilson et al. (1995) proposed that olivine melilitites represent characteristic near-primary partial melts of the thermal boundary layer at the base of the lithosphere. The deep Saxothuringian-Moldanubian contact in the Bohemian Massif is marked by a lithospheric thinning to about $80-90$ $\mathrm{km}$ beneath the (western) Ohře Rift (Babuška and Plomerová 2010; Geissler et al. 2010). According to the model of the Hessian Depression of Wedepohl (1987) the olivine nephelinite/melilitite magma was generated by partial melting at similar depths of c. $90 \mathrm{~km}$. The

Fig. 7 Rocks of the melilitic rock series of the Ploučnice River Region in the $\mathrm{CaO} / \mathrm{MgO}$ vs. $\mathrm{SiO}_{2} / \mathrm{Al}_{2} \mathrm{O}_{3}$ diagram after Gudfinnson and Presnall (2005) and the compositional ranges of experimental melts at various pressures and volatile conditions. The experimental compositions of melilitic glasses of Dasgupta et al. (2007) and natural melilitic rocks from Madagascar (Melluso et al. 2011) are shown for comparison. Data from Ulrych et al. (2008) and this work. 
Tab. $5 \mathrm{Rb}-\mathrm{Sr}$ and $\mathrm{Sm}-\mathrm{Nd}$ isotopic data for camptonite-monchiquite "wesselite" dykes.

\begin{tabular}{|c|c|c|c|c|c|c|c|c|c|c|c|c|c|c|}
\hline Sample & Locality & Rock & $\begin{array}{l}\text { Age } \\
\text { (Ma) }\end{array}$ & $\begin{array}{c}\mathrm{Rb} \\
(\mathrm{ppm})\end{array}$ & $\begin{array}{c}\mathrm{Sr} \\
(\mathrm{ppm})\end{array}$ & ${ }^{87} \mathrm{Rb} /{ }^{86} \mathrm{Sr}$ & ${ }^{87} \mathrm{Sr} /{ }^{86} \mathrm{Sr}(\mathrm{m})$ & ${ }^{87} \mathrm{Sr} /{ }^{86} \mathrm{Sr}(\mathrm{t})$ & $\begin{array}{c}\mathrm{Nd} \\
(\mathrm{ppm})\end{array}$ & $\begin{array}{c}\mathrm{Sm} \\
(\mathrm{ppm})\end{array}$ & ${ }^{147} \mathrm{Sm} /{ }^{144} \mathrm{Nd}$ & ${ }^{3} \mathrm{~N}$ & ${ }^{143} \mathrm{Nd}$ & $\varepsilon_{\mathrm{Nd}}(\mathrm{t})$ \\
\hline 49 & Veselí & monchiquite & 30.9 & 133 & 725 & 0.531 & $0.704826 \pm 10$ & 0.7046 & 47 & 8.6 & 0.1102 & $0.512793 \pm 7$ & 0.512771 & 3.5 \\
\hline $1 / 13$ & Veselí & monchiquite & 30.9 & 154 & 854 & 0.522 & $0.706082 \pm 13$ & 0.7061 & 51 & 9.3 & 0.1112 & $0.512791 \pm 6$ & 0.512769 & 3.3 \\
\hline
\end{tabular}

origin of the melilitic magma characterized by extreme silica undersaturation and high $\mathrm{Ca}$ content probably involved incongruent melting of clinopyroxene in presence of substantial amounts of $\mathrm{H}_{2} \mathrm{O}$ and $\mathrm{CO}_{2}$ at $\mathrm{P}>26 \mathrm{kbar}$ (Morgan et al. 1985).

The principal role of $\mathrm{CO}_{2}$ in the genesis of melilitic volcanic rocks has been established by Brey et al. (1978). The release of $\mathrm{CO}_{2}$ during magma ascent, due to its wellknown low solubility at low pressures, could account for the geochemistry, mineralogy and physical properties of such magmas (Melluso et al. 2011). A comparison between the bulk-rock compositions and experimental results in the $\mathrm{CaO}-\mathrm{MgO}-\mathrm{Al}_{2} \mathrm{O}_{3}-\mathrm{SiO}_{2}-\mathrm{CO}_{2}$ system points to a similarity with near-solidus melts in a moderately $\mathrm{CO}_{2}$-rich garnet-bearing mantle at $\sim 3 \mathrm{GPa}$ (Fig. 7 - Gudfinnson and Presnal 2005). The experimental results indicate a likely depth of melting of at least 90-105 $\mathrm{km}$ to produce the variety of magmas. This corresponds with the estimated depths of $c .80-90 \mathrm{~km}$ of the mantle lithosphere base beneath the Ohře Rift in the western part of the Bohemian Massif (Babuška and Plomerová 2010). Melilitic rocks from Madagascar (Melluso et al. 2011) and experimental melilitite glasses of Dasgupta et al. (2007) share the similar position in Fig. 7 with moderately $\mathrm{CO}_{2}$-rich conditions.

The $\mathrm{K}$ troughs in primitive mantle-normalized diagrams in Fig. 5 are significant in the interpretation of magma sources (see Melluso et al. 2011 and citations therein). Le Roex et al. (2003) ascribed this feature to the presence of amphibole or phlogopite as residual phases during low-degree melting, characteristic in particular of melilitic rocks (kimberlites).

Variations in the chemical and isotopic compositions of melilitic magmas of northern Bohemia are related to lithospheric thinning or incipient rifting (Ulrych et al. 2006) - the pre-rift period of Ulrych et al. (2008) - and point to local differences in their sources. The primary mineral association of olivine + melilite + spinels \pm clinopyroxene is universal and characteristic of ultramafic melilitic rocks of the Osečná Complex and the associated Devil's Walls swarm.

Negligible, if any, fractionation can be inferred from the high $\mathrm{Mg} \#$ values of 73-81 for the melilitic rock series (Ulrych et al. 2008 and new data). Only olivine melilitolite of the Osečná Complex was substantially affected by late-magmatic crystallization and postmagmatic fluids, causing metasomatic transformation (see Section 5.1). Glimmerites with $\mathrm{Mg} \#$ of $74-76$ are chemically similar to the parental olivine melilitolite while pegmatites $(\mathrm{Mg} \#$ =64-66) and ijolites $(\mathrm{Mg} \#=64)$ represent evolved metasomatic products enriched in incompatible elements (Ulrych et al. 2008).

The Nd-Sr isotopic data of the melilitic rock series of Ulrych et al. (2008) indicate similar, yet heterogeneous, mantle sources particularly with respect to ${ }^{87} \mathrm{Sr} /{ }^{86} \mathrm{Sr}$ ratios ( $\sim 0.7033$ to $\sim 0.7049)$. An alternative interpretation of the relatively high initial ${ }^{87} \mathrm{Sr} /{ }^{86} \mathrm{Sr}$ ratios is a late- and/or postmagmatic alteration of the rocks, lowering their $\mathrm{Rb} / \mathrm{Sr}$ ratios. The high positive initial $\varepsilon_{\mathrm{Nd}}$ values $(+3.2$ to +5.2$)$ are interpreted to indicate melting of depleted, although moderately heterogeneous mantle sources precluding significant melt contamination by evolved continental crust. New Nd-Sr data on the monchiquite-camptonite rock series (Tab. 5) show a fair variation in initial ${ }^{87} \mathrm{Sr} /{ }^{86} \mathrm{Sr}$ ratios ( $\sim 0.7046$ to $\sim 0.7061)$ corresponding most probably to a substantial postmagmatic alteration of the dykes, nevertheless a minor crustal contamination cannot be excluded. The positive initial $\varepsilon_{\mathrm{Nd}}$ values ( +3.3 to +3.5$)$ correspond well to those known from the melilitic rock series.

The presence of monticellite in silica-poor polzenite of the Vesec type and olivine micro-melilitolites of the PRR indicates a transition toward more silica-undersaturated compositions, where clinopyroxene tends to be minor or absent, due to the low silica activity of the primitive magmas characterized by higher Mg\# (Melluso et al. 2011).

Rocks of the monchiquite-camptonite dyke series show signs of fractionation. Presence of phenocrysts of kaersutite and phlogopite in this series indicates that the primary magma was enriched in $\mathrm{H}_{2} \mathrm{O}$ and other volatile components but impoverished in incompatible elements compared to the melilitic rock series.

The whole-rock major- and trace-element contents imply that the rocks of melilitic dyke association were derived from subcontinental lithospheric mantle sources. The contents of incompatible trace elements in the melilitic rock series are high and variable $(\mathrm{Sr}=810-2200$ ppm, $\mathrm{Ba}=630-2100 \mathrm{ppm}$ ), significantly higher than those of average continental crust $(\mathrm{Sr} \sim 320 \mathrm{ppm}, \mathrm{Ba} \sim$ 460 ppm; Rudnick and Gao 2003).

The OIB-like incompatible trace-element ratios, such as high $\mathrm{Nb} / \mathrm{U}(32-68)$, low $\mathrm{La} / \mathrm{Nb}(0.4-1.0)$ and variable $\mathrm{Ba} / \mathrm{Nb}$ (4-12), suggest a limited lithospheric mantle contamination (see Hofmann 1988; Sun and McDonough 1989; Melluso et al. 2011). For the generation of such a melt it is necessary to speculate on an upper mantle source enriched in incompatible elements, together with 
a small degree of partial melting of the source material. The minor positive $\mathrm{Nb}$ anomaly both in the melilitic and monchiquite-camptonite rock series might indicate $\mathrm{Nb}$ fractionation during the passage of primary asthenospheric magma through a metasomatized lithospheric mantle (Hofmann et al. 1986).

Evidence for such metasomatized upper mantle may be provided by xenoliths of glimmerite to mica clinopyroxenite in the polzenite dykes. To the contrary, dunite to harzburgite xenoliths in melilite-bearing olivine nephelinite most likely represent a depleted mantle which underwent moderate to high-degree partial melting. Garnet peridotite, eclogite, norite and ferro-dunite xenoliths are entrained from the local crystalline basement and occur in vent breccias only (Ulrych et al. 2000c). High Ca contents in the melilitic rocks point to a clinopyroxene-rich veined mantle sensu Lloyd et al. (1991).

Rocks of the monchiquite-camptonite series, in comparison to the melilitic rock series, are characterized by low contents of compatible elements such as $\mathrm{Ni}(\sim 20-110$ $\mathrm{ppm})$, Co $(\sim 30-50 \mathrm{ppm}), \mathrm{Sc}(\sim 20-50 \mathrm{ppm})$ and $\mathrm{Cr}$ $(\sim 70-260 \mathrm{ppm})$. The contents of incompatible elements, e.g. $\mathrm{Sr}(\sim 700-1000 \mathrm{ppm})$ and $\mathrm{Ba}(\sim 600-1100 \mathrm{ppm})$ in the rocks of this series are high and variable, though lower than those in the melilitic rock series. Chemical analyses of the "wesselite" from the original locality demonstrate an inhomogeneity and probably a zoned development of the dyke and/or a presence of several dykes (Tab. 2). The incompatible element ratios and the $\mathrm{Nb}$ anomaly are largely similar to those of the melilitic rocks series.

The presence of two age-contrasting melilitic (Late Cretaceous to Palaeocene) and tephrite-basanitic (Oligocene) series in northern Bohemia has no analogy either in the Bohemian Massif (Ulrych et al. 2011) or elsewhere in the Circum-Mediterranean anorogenic Cenozoic igneous province of Lustrino and Wilson (2007).

\section{Conclusions}

- The Cenozoic Central European Volcanic Province includes two main diachronous volcanic rock series in the northern part of the Bohemian Massif: (i) the Late Cretaceous to Early Tertiary ultramafic melilitic rock series including ultramafic clinopyroxene-free lamprophyres - polzenites (80-61 Ma), and (ii) the Oligocene monchiquite-camptonite dyke series (31-28 Ma) free of melilite, belonging to the tephrite-basanite rock series, which was erroneously genetically ascribed to the melilitic series.

- Rare occurrences of ultramafic melilitic and melilite-bearing rocks concentrate to the Osečná Complex associated with the Devil's Walls swarm. These rock suites occur in the outer parts of the Ohře/Eger Rift at the junction with the Lusatian Fault Zone in northern Bohemia.

- Olivine melilitolite of the Osečná intrusion with a marginal facies of micro-melilitolite are porphyritic rocks with attributes of lamprophyres (textural characteristics, presence of phlogopite, carbonate, and richness in incompatible elements). Similar modal and chemical compositions of the Vesec type of polzenite dykes and micro-melilitolite of the Osečná intrusion correspond fully to the concept of the lamprophyric facies. Nevertheless, polzenites share all characteristics quoted for (ultramafic) lamprophyres in the literature.

- The steeply dipping dykes associated with the Osečná intrusion are composed of a melilitic ultramafic lamprophyre with volatile-rich mineral association polzenite. Clinopyroxene-free polzenites of the Vesec type are micro-porphyritic, with phenocrysts being represented by olivine with monticellite rims set in groundmass with poikilitic phlogopite and abundant perovskite. Polzenites of the Modlibohov type are rich in phlogopite, zoned foid of haüyne-sodalite composition, poor in olivine with minor clinopyroxene content, passing to the (clinopyroxene-rich) alnöite of the Luhov type. Carbonates are present in variable amounts (1-10 vol. \%) in all types of melilitic rocks.

- The Devil's Walls swarm is temporally, spatially and genetically associated with the Osečná Complex. Steeply dipping dykes consist predominantly of melilite-bearing olivine nephelinite with rare transitions to olivine melilitite.

- Melilitic and melilite-bearing rocks of the PRR show very high Mg\# (73-81). Polzenites of the Vesec type and olivine micro-melilitolites have mean values of 78 , thus corresponding rather to mafic cumulates. Peridotite xenoliths are present in all these rocks. Glimmerite to mica clinopyroxenite xenoliths in polzenites represent samples of a metasomatized upper mantle.

- Geological, mineralogical, petrological and geochemical evidence from the melilitic rock suite points to the presence of a singular rock series originated from a common mantle magma source. They can be interpreted as low-degree partial melts of a heterogeneous, clinopyroxene-veined mantle re-fertilized by metasomatic phlogopite \pm dolomite and other phases rich in incompatible elements. Mantle metasomatism was probably related to carbonatitic magmatism associated with incipient Neoidic rifting of the lithosphere in the northern and northwestern parts of the Bohemian Massif.

Acknowledgements. This research was financially supported by institutional project RVO 67985831 of the Institute of Geology AS CR, v. v. i. and the Grant Agency of the Academy of Sciences of the Czech Republic, 
project IAA300130902. The work was also financed by the project "EXCELLENT TEAMS" at Brno University of Technology CZ.1.07/2.3.00/30.0005. The authors thank A. Paluska for making access to historical sites of polzenite rocks, sampling and permission to publish nine chemical analyses financed by A. Paluska and the DIAMO state enterprise, Stráž p. R. Thanks are also due to V. Vonásková with P. Povondra for whole-rock major-element analyses and L. Strnad for ICP-MS analyses (all Faculty of Science, Charles University, Prague), J. Durišová (Institute of Geology AS CR, v.v.i., Prague) for ICP-MS analyses and E. Hegner (Universität München) for $\mathrm{Sr}-\mathrm{Nd}$ isotopic data. We are indebted to J. Pavková and J. Rajlichová for technical assistance and to reviewers J. Lindline and E.A. Chernysheva for their comments and improvements of the manuscript. The manuscript was considerably improved by the guest editor V. Rapprich and the journal editor V. Janoušek.

\section{References}

Abratis M, Munsel D, Viereck-Götte L (2009) Melilithite und Melilith-führende Magmatite des sächsischen Vogtlands: Petrographie und Mineralchemie. Z geol Wiss 37: 41-79

Adamovič J, Coubal M (1999) Intrusive geometries and Cenozoic stress history of the northern part of the Bohemian Massif. Geolines 9: 5-14

Alibert C, Leterrier R, Albarède F (1983) The transition from alkali basalts to kimberlites: isotope and trace element evidence from melilitites. Contrib Mineral Petrol 82: $176-186$

BabušKa V, Plomerová J (2010) Mantle lithosphere control of crustal tectonics and magmatism of the western Ohře (Eger) Rift. J Geosci 55: 171-186

Bailey K, Lloyd F, Kearns S, Stoppa F, Eby N, Woolley A (2005) Melilitite at Fort Portal, Uganda: another dimension to the carbonate volcanism. Lithos 85: 15-25

BALOGH K (1985) K/Ar dating of Neogene volcanic activity in Hungary. Experimental technique, experience and methods of chronological studies. Unpublished ATOMKI Report D/1, Debrecen, pp 277-278

Barbieri M, Ghiara Mr, Stanzione D, Viklar LM, Pezzuti NE, Segal SJ (1997) Trace-element and isotope constraints on the origin of ultramafic lamprophyres from Los Alisos (Sierra Subandinas, Northern Argentina). J South Amer Earth Sci 10: 39-47

Beard AD, Downes H, Hegner E, Sablukov SM, Vetrin VR, BALOGH K (1998) Mineralogy and geochemistry of Devonian ultramafic minor intrusions of the southern Kola Peninsula, Russia: implication for the petrogenesis of kimberlites and melilitites. Contrib Mineral Petrol 130: $288-303$
Bernstein S, Leslie AG, Higgins AK, Brooks CK (2000) Tertiary alkaline basic volcanics in the Nunatak Region, North-East Greenland: new discoveries and their similarities to the maymechites of Siberia. Lithos 53: 1-20

Boynton WV (1984) Cosmochemistry of the rare earth elements: meteorite studies. In: HeNDERSON P (ed) Rare Earth Element Geochemistry. Elsevier, Amsterdam, pp 63-114

Brey G (1978) Origin of olivine melilitites - chemical and experimental constraints. J Volcanol Geotherm Res 3: 61-88

Chauvel C, Hofmann AW, Vidal P (1992) HIMU-EM: the French Polynesian connection. Earth Planet Sci Lett 110: 99-119

Dasgupta R, Hirschmann MM, Smith ND (2007) Partial melting experiments of peridotite $+\mathrm{CO}_{2}$ at $3 \mathrm{GPa}$ and genesis of ocean islands basalts. J Petrol 47: 647-671

Dawson JB, Smith JV, Jones AP (1985) A comparative study of bulk rock and mineral chemistry of olivine melilitites and associated rocks from East and South Africa. Neu Jb Mineral, Abh 152: 143-175

Di Batistini G, Montanini A, Vernia L, Venturelli G, TONARINI S (2001) Petrology of melilite-bearing rocks from Montefiascone Volcanic Complex (Roman Magmatic Province): new insight into the ultrapotassic volcanism of Central Italy. Lithos 59: 1-24

Dunworth EA, Wilson M (1998) Olivine melilitites of the SW German Tertiary volcanic province: mineralogy and petrogenesis. J Petrol 39: 1805-1836

ECKERMANN H VON (1961) The petrogenesis of the Alnö alkaline rocks. Bull Geol Inst Univ Uppsala 40: 25-36

Egorov LS (1970) Carbonatites and ultrabasic-alkaline rocks of the Maimecha-Kotui region, N. Siberia. Lithos 3: 341-359

Francis EH (1982) Magma and sediment - I. Emplacement mechanism of late Carboniferous tholeiite sills in northern Britain. J Geol Soc, London 139: 1-20

Frey F, Green DH, Roy SD (1978) Integrated models of basalt petrogenesis: a study of quartz tholeiites to olivine melilitites from southeastern Australia utilizing geochemical and experimental petrological data. J Petrol 19: 463-513

Geissler W H, SOdoudi F, Kind R (2010) Thickness of the central and eastern European lithosphere as seen by S receiver functions. Geophys J Int 181: 604-634

Gudfinnson GH, Presnal DC (2005) Continuous gradations among primary carbonatitic, kimberlitic, melilititic, basaltic, picritic and komatiitic melts in equilibrium with garnet lherzolite at 3-8 GPa. J Petrol 46: 1645-1659

Hamois L, Mineau R (1991) Geochemistry of the Île Cadieux monticellite alnöite, Quebec, Canada. Can J Earth Sci 28: 1050-1057 
Hegner E, Vennemann TW (1997) Role of fluids in the origin of Tertiary European intraplate volcanism: evidence from $\mathrm{O}, \mathrm{H}$, and Sr isotopes in melilitites. Geology 25: 1035-1038

Hegner E, Walter H-J, Satir M (1995) Pb-Sr-Nd isotopic compositions and trace element geochemistry of megacrysts and melilitites from the Urach volcanic field: source composition of small volume melts under SW Germany. Contrib Mineral Petrol 122: 322-335

Hoernle K, Schmincke H-U (1993) The petrology of the tholeiites through melilite nephelinites on Grand Canaria, Canary Islands: crystal fractionation, accumulation, and depths of melting. J Petrol 34: 573-597

Hofmann AW (1988) Chemical differentiation of the Earth: the relationship between mantle, continental crust, and the oceanic crust. Earth Planet Sci Lett 90: 297-314

Hofmann AW, Jochum KP, Seufert M, White WM (1986) $\mathrm{Nb}$ and $\mathrm{Pb}$ in oceanic basalts: new constraints on mantle evolution. Earth Planet Sci Lett 79: 33-45

Ishikawa A, Maruyama S, Komiya T (2004) Layered lithosphere mantle beneath the Ontong Java Plateau: implications from xenoliths in alnöite, Malaita, Solomon Islands. J Petrol 45: 2011-2044

JACOBSEn SB, WASSERburG GJ (1980) Sm-Nd isotopic evolution of chondrites. Earth Planet Sci Lett 50: 139-155

Jochum KP, Nohl U (2008) Reference materials in geochemistry and environmental research and the GeoReM database. Chem Geol 253: 50-53

Johannsen A (1949) A Descriptive Petrography of the Igneous Rocks IV. University Press, Chicago, pp 1-523

Johnson WM, Maxwell JA (1981) Rock and Mineral Analysis. Wiley, New York, pp 1-489

KelLer J (1984) Der jungtertiäre Vulkanismus Südwestdeutschlands: Exkursionen in Kaiserstuhl und Hegau. Fortschr Mineral 62: 2-35

Keller J, Brey G, Lorenz V, Sachs P (1990) Pre-conference excursion 2A: Volcanism and petrology of the Upper Rhinegraben (Urach-Hegau-Kaiserstuhl). IAVCEI International Volcanological Congress, Mainz 1990, pp 1-31

Keller J, Zaytsev A, Wiedenmann D (2006) Primary magmas at Oldoinyo Lengai: the role of olivine melilitites. Lithos 91: 150-172

KRÜGER JC, Romer RL, KÄMPF H (2013) Late Cretaceous ultramafic lamprophyres and carbonatites from the Delitzsch Complex, Germany. Chem Geol 353: 140-150

KüHn P (1999) The "Střelnice" dyke in Doksy (north Bohemia). Bull mineral-petrolog Odd Nár Muz (Praha) 7: 169-172

Le BAs MJ (1989) Nephelinitic and basanitic rocks. J Petrol 30: 1299-1312

Le Maitre RW (1989) A Classification of Igneous Rocks and Glossary of Terms. Blackwell Scientific, Oxford, pp 1-193

Le Maitre RW (2002) Igneous Rocks. A Classification and Glossary of Terms. $2^{\text {nd }}$ Edition. Cambridge University Press, Cambridge, pp 1-236
Le Roex AP, Bell DR, Davis, P (2003) Petrogenesis of group I kimberlites from Kimberley, South Africa: evidence from bulk-rock chemistry. J Petrol 44: 2261-2286

Lloyd FE, Huntingdon AT, Davies GR, Nixon PH (1991) Phanerozoic volcanism of southwest Uganda. A case for regional $\mathrm{K}$ and LILE enrichment of the lithosphere beneath a domed and rifted continental plate. In: KAMPUNZU AB, LubALA RT (eds) Magmatism in Extensional Structural Settings. Springer, Berlin, pp 23-72

Lustrino M, Wilson M (2007) The Circum-Mediterranean anorogenic Cenozoic igneous province. Earth Sci Rev 81: $1-65$

Maaloe S, James D, Smedley P, Petersen S, Garmann LB (1992) The Koloa volcanic suite of Kaunai, Hawaii. J Petrol 33: 761-784

Melluso LM, Le Roex AP, Morra VM (2011) Petrogenesis and Nd-, Pb-, Sr-isotope geochemistry of the Cenozoic olivine melilitites and olivine nephelinites ("ankaratrites") in Madagascar. Lithos 127: 505-521

Mitchell RH (1994) The lamprophyre facies. Mineral Petrol 51: 137-146

Morgan JW, Czamanske GK, Wandless GA (1985) Origin and evolution of the alkalic ultramafic rocks in the Coyote Peak diatreme, Humboldt County, California. Geochim Cosmochim Acta 49: 749-759

ODIN GS AND 35 AUTHORS (1982) Interlaboratory standards for dating purposes. In: ODIN GS (ed) Numerical Dating in Stratigraphy. Wiley \& Sons, Chichester, pp 123-149

Pearce NGJ, Leng MJ, Emeleus CH, Bedford CM (1997) The origins of carbonatites and related rocks from the Gronnedal-Ika nepheline syenite complex, South Greenland: C-O-Sr isotope evidence. Mineral Mag 61: 515-529

Pfeiffer L (1978) Beitrag zur Petrochemie der sächsischen Tertiärvulkanite. Freiberg Forsch H C 333: 1-64

Pfeiffer L (1994) Der Tertiäre Magmatismus im Erzgebirge und in den benachbarten Gebieten der Tschechischen Republik. Beih Eur J Mineral 6: 179-228

Piromallo C, Gasperini D., Macera P, Faccenna C (2008) A late Cretaceous contamination episode of the EuropeanMediterranean mantle. Earth Planet Sci Lett 268: 15-27

Pivec E, Povondra P, Rutšek J, Ulrych J (1986) Petrology and geochemistry of the Osečná intrusion in the Ještěd foothills (Northern Bohemia). Acta Montana 74: 23-31

Pivec E, Ulrych J, HöHndorf A, Rutšek J (1998) Melilitic rocks from northern Bohemia: geochemistry and mineralogy. Neu Jb Mineral, Abh 1998: 312-339

PotTs PJ (1995) A Handbook of Silicate Rock Analysis. Blackie Academic and Professional, London, pp 1-622

Prodehl C, Mueller S, Haak V (1995) The European Cenozoic rift system. In: Olsen KH (ed) Continental Rifts: Evolution, Structure, Tectonics. Developments in Geotectonics 25: Elsevier, Amsterdam, pp 133-212 
RASS IT (2008) Melilite-bearing and melilite-free rock series in carbonatite complexes: derivatives from separate primitive melts. Canad Mineral 46: 951-969

Renno AD, Stanek KP, Lobst R, Pushkarev Y (2003a) A new lamprophyre species from the Klunst quarry (Ebersbach, Lusatia, Germany) - geochemical and petrological implications. Z geol Wiss 31: 1-20

Renno AD, Hacker BR, Stanek KP (2003b) An Early Cretaceous (126 Ma) ultramafic alkaline lamprophyre from the Quarry Klunst (Ebersbach, Lusatia, Germany). Z geol Wiss 31: 31-36

Rhodes JM (1981) Characteristics of primary basaltic magmas. In: Basaltic Volcanism Study Project: Basaltic Volcanism of the Terrestrial Planets. Pergamon Press, New York, pp 128-147

Rudnick RL, GaO S (2003) Composition of the continental crust. In: Rudnick, RL (ed) The Crust. Treatise on Geochemistry 3: Elsevier-Pergamon, Oxford, pp 1-64

Rock NMS (1987) The nature and origin of lamprophyres: an overview. In: Fiton JG, Upton BGJ (eds) Alkaline Igneous Rocks. Geological Society London Special Publications 30: 191-226

Rock NMS (1991) Lamprophyres. Blackie and Sons Ltd., Glasgow and London, pp 1-285

Rosenbusch H (1887) Mikroskopische Physiographie der Mineralien und Gesteine. Vol. II Massige Gesteine, $2^{\text {nd }}$ edition. Schweizerbart'sche Verlag, Stuttgart, pp 1-877

Rosenbusch H (1907) Mikroskopische Physiographie der Mineralien und Gesteine. Vol II Massige Gesteine, $4^{\text {th }}$ edition. Schweizerbart'sche Verlag, Stuttgart, pp $1-716$

Scheumann KH (1913) Petrographische Untersuchungen an Gesteinen des Polzengebietes in Nord-Böhmen. Abh kön Sächs Gesel Wiss math-phys K1 32: 607-776

Scheumann KH (1922) Zur Genese alkalisch-lamprophyrischen Ganggesteine. Zbl Mineral Geol Paläontol 2: 495-545

SEIfERt W, Büchner J, Tietz O (2008) Der „Melilithbasalt“ von Görlitz im Vergleich mit dem Melilithitit vom Zeughausgang: Retrospektive und neue mineral-chemische Ergebnisse. Z geol Wiss 36: 155-176

SöLlnER J (1913) Über den Bergalith, ein neues melilitreiches Ganggestein aus dem Kaiserstuhl. Mitt Badisch geol Landesanst 7: 2

StÖRmer JC, Nicholls J (1978) XLFRAC: a program for interactive testing of magmatic differentiation models. Comput and Geosci 87: 51-64

Streckeisen A (1979) Classification and nomenclature of volcanic rocks, lamprophyres, carbonatites and melilitic rocks. Neu Jb Mineral, Abh 134: 1-14

Strnad L, Mihaljevič M, ŠEBeK O (2005) Laser ablation and solution ICP-MS determination of rare earth elements in USGS BIR-1G, BHVO-2G and BCR-2G glass reference material. Geostand Geoanal Res 29: 303-314
Sun SS, McDonough WF (1989) Chemical and isotopic systematics of oceanic basalts: implications for mantle compositions and processes. In: SAUNDERS AD, Norry MJ (eds) Magmatism in the Ocean Basins. Geological Society London Special Publications 42: 313-345

Tappe S, Foley SF, Jenner GA, Kuarsgaard BJ (2005) Integrating ultramafic lamprophyres into the IUGS classification of igneous rocks: rationale and implications. J Petrol 46: 1893-1900

TRÖGER WE (1939) Über Theralith and Monchiquit. Zbl Miner Geol Paläont Abt A: 80-94

Ulrych J, Povondra P, Rutšek J, Pivec E (1988) Melilitic and melilite-bearing subvolcanic rocks from the Ploučnice river region, Czechoslovakia. Acta Univ Carol, Geol 195-231

Ulrych J, Povondra P, Huspeka J, Pivec E, Rutšek J (1990) Chemical composition of melilitic volcanics of northern part of the Bohemian Massif. Report of the Projects II-41/07 and II/4/4/05. Institute of Geosciences, Faculty of Science, Charles University, Prague, pp 1-123 (in Czech)

Ulrych J, Pivec E, Povondra P, Rutšek J (1991) Rockforming minerals of polzenite and cognate melilitic rocks from northern Bohemia, Czechoslovakia. Acta Univ Carol, Geol Fediuk Vol 2: 139-163

Ulrych J, Pivec E, ŽÁK K, Bendl J, BosÁk P (1993) Alkaline and ultramafic carbonate lamprophyres in Central Bohemian Carboniferous basins, Czech Republic. Mineral Petrol 48: 65-81

Ulrych J, Povondra P, Pivec E, Rutšek J, Bendl J, Bilik I (1996) Alkaline ultramafic sill at Dvůr Králové nad Labem, Eastern Bohemia: petrological and geochemical constraints. Acta Univ Carol, Geol 53-79

Ulrych J, Pivec E, Lang M, Lloyd FE (2000a) Ijolitic segregations in melilite nephelinite of Podhorní vrch volcano, Western Bohemia. Neu Jb Mineral, Abh 175: 317-348

Ulrych J, Cajz V, Pivec E, Novák JK, Nekovařík Č, Balogh $\mathrm{K}$ (2000b) Cenozoic intraplate alkaline volcanism of Western Bohemia. Stud Geophys Geod 44: 346-351

Ulrych J, Pivec E, Povondra P, RutšEk J (2000c) Uppermantle xenoliths in melilitic rocks of the Osečná Complex, North Bohemia. J Czech Geol Soc 45: 79-93

Ulrych J, Svobodová J, Balogh K (2002) The source of Cenozoic volcanism in the České Středohoří Mts., Bohemian Massif. Neu Jb Mineral, Abh 177: 133-162

Ulrych J, Lloyd Fe, Balogh K, Hegner E, Langrová A, LANG M, NovÁK JK, ŘAnda Z (2005) Petrogenesis of alkali pyroxenite and ijolite xenoliths from the Tertiary Loučná-Oberwiesenthal Volcanic Centre, Bohemian Massif in the light of new mineralogical, geochemical and isotopic data. Neu Jb Mineral, Abh 182: 57-79

Ulrych J, Pešek J, ŠtěPÁnková-Svobodová J, Bosák P, Lloyd FE, von Seckendorff V, Lang M, Novák JK (2006) Permo-Carboniferous volcanism in late Variscan continental basins of the Bohemian Massif (Czech 
Republic): geochemical characteristic. Chem Erde 66: 37-56

Ulrych J, Dostal J, Hegner E, Balogh, K, Ackerman L (2008) Late Cretaceous to Paleocene melilitic rocks of the Ohře/Eger Rift in northern Bohemia, Czech Republic: insights into the initial stages of continental rifting. Lithos 101: 141-161

Ulrych J, Jelínek E, Řanda Z, Lloyd FE, Balogh K, HegNER E, NovÁK JK (2010) Geochemical characteristics of the high- and low-Ti basaltic rocks from the uplifted shoulder of the Ohře (Eger) Rift, Western Bohemia. Chem Erde 70: 319-333

Ulrych J, Dostal J, Adamovič J, Jelínek E, Špaček P, HegNER E, BALOGH K (2011) Recurrent Cenozoic volcanic activity in the Bohemian Massif (Czech Republic). Lithos 123: $133-144$

WeDEPOHL KH (1987) Kontinentaler Intraplaten-Vulkanismus am Beispiel der tertiären Basalte der Hessischen Senke. Fortschr Mineral 65: 19-47

WiLKINSON JFG, Stolz AJ (1983) Low-pressure fractionation of strongly undersaturated alkaline ultrabasic magma: the olivine-melilite-nephelinite at Moiliili, Oahu, Hawaii. Contrib Mineral Petrol 83: 363-374
Wilson M, Rosenbaum JM, Dunworth EA (1995) Melilitites: partial melts of the thermal boundary layer? Contrib Mineral Petrol 119: 181-196

WimMENAUER W (1973) Lamprophyre, Semilamprophyre und anchibasaltische Ganggesteine. Fortschr Mineral 51: 3-67

Woolley AR, Bergman CC, Edgar AD, le Bas MJ, Mitchell RH, Rock NMS, Scott-Smith BH (1996) Classification of lamprophyres, lamproites, kimberlites, and the kalsilitic, melilitic and leucitic rocks. Canad Mineral 34: 175-186

WURm F (1883) Über das Vorkommen von Melilithbasalt zwischen Böhmisch-Leipa und Böhmisch-Aicha. SitzBer Kön Böhm Gesell Wiss (Prag) 2: 1-8

Wurm F (1884) Die Teufelsmauer zwischen Oschitz und Böhm.-Aicha. Nordböhmischer Excursions-Club, Česká Lípa, pp 1-35

ZAHÁLKA B (1905) Eruptive rocks from the Mělník and Mšeno neighbourhoods. Věst Král České Spol Nauk v Praze III: 1-79 (in Czech with German abstract)

Zhabin AG, Surina NP (1970) Petrology of dikes, sills and pipes of the Maymecha-Kotuyi Province. Nauka, Moscow, pp 1-202 (in Russian) 